\title{
Testing scalar versus vector dark matter
}

\author{
Duarte Azevedo, ${ }^{2, *}$ Mateusz Duch, ${ }^{1, \dagger}$ Bohdan Grzadkowski, ${ }^{1, \star}$ Da Huang, ${ }^{1, \S}$ Michal Iglicki, ${ }^{1, \|}$ and Rui Santos ${ }^{2, \boldsymbol{f}}$ \\ ${ }^{1}$ Faculty of Physics, University of Warsaw, Pasteura 5, 02-093 Warsaw, Poland \\ ${ }^{2}$ Centro de Física Teórica e Computacional, Faculdade de Ciências, Universidade de Lisboa, \\ Campo Grande, Edifício C8 1749-016 Lisboa, Portugal
}

(Received 24 September 2018; published 9 January 2019)

\begin{abstract}
We investigate and compare two simple models of dark matter (DM): a vector and a scalar DM model. Both models require the presence of two physical Higgs bosons $h_{1}$ and $h_{2}$ which come from mixed components of the standard Higgs doublet $H$ and a complex singlet $S$. In the vector model, the extra $U(1)$ symmetry is spontaneously broken by the vacuum of the complex field $S$. This leads to a massive gauge boson $X^{\mu}$ that is a DM candidate stabilized by the dark charge conjugation symmetry $S \rightarrow S^{*}, X^{\mu} \rightarrow-X^{\mu}$. On the other hand, in the scalar model the gauge group remains the standard one. The DM field $A$ is the imaginary component of $S$ and the stabilizing symmetry is also the dark charge conjugation $S \rightarrow S^{*}$ $(A \rightarrow-A)$. In this case, in order to avoid spontaneous breaking, the $U(1)$ symmetry is broken explicitly, but softly, in the scalar potential. The possibility to disentangle the two models has been investigated. We have analyzed collider, cosmological, DM direct and indirect detection constraints and shown that there are regions in the space spanned by the mass of the nonstandard Higgs boson and the mass of the DM particle where the experimental bounds exclude one of the models. We have also considered possibility to disentangle the models at $e^{+} e^{-}$collider and concluded that the process $e^{+} e^{-} \rightarrow Z+$ DM provides a useful tool to distinguish the models.
\end{abstract}

DOI: 10.1103/PhysRevD.99.015017

\section{INTRODUCTION}

The Higgs boson was discovered at CERN's Large Hadron Collider (LHC) by the ATLAS [1] and CMS [2] collaborations thus turning one important page in our knowledge of the Universe by not only discovering a new particle but also to hint very strongly that there is a mechanism of spontaneous symmetry breaking giving mass to both gauge bosons and fermions. Over the years, it has become increasingly clear that this boson resembles very much the one predicted by the standard model (SM). However, there are still many unsolved problems in particle physics that are not answered by the SM. One of them is the existence of the dark matter (DM) in the universe which presence cannot be attributed to any known particles.

\footnotetext{
*dazevedo@alunos.fc.ul.pt mateusz.duch@fuw.edu.pl

tbohdan.grzadkowski@fuw.edu.pl

$\S$ da.huang@fuw.edu.pl

"Michal.Iglicki@fuw.edu.pl

"rasantos@fc.ul.pt
}

Published by the American Physical Society under the terms of the Creative Commons Attribution 4.0 International license. Further distribution of this work must maintain attribution to the author(s) and the published article's title, journal citation, and DOI. Funded by SCOAP ${ }^{3}$.
Although the measurements of the Higgs couplings are quite demanding for the so-called beyond the standard model (BSM) models, there is still plenty of space in the present results to include new physics. Some of the BSM models can be compatible with the measurements while providing solutions to some of the outstanding questions of particle physics. Such is the case of the models studied in this work. Both the extension with a complex singlet [3-8] and the extension with a new Abelian vector boson together with a complex singlet [9-14] provide DM candidates still compatible with collider bounds, and direct or indirect detection experiments. The models can also undergo a strong first-order phase transition during the era of EWSB [7,15-23] thus explaining electroweak baryogenesis.

Extra scalar singlets are dimension one fields and therefore prone to couple to the SM scalar sector in a renormalizable way without any suppression by inverse powers of the scale of BSM, a concept introduced in [24] and known as the Higgs portal. Assuming the scale of new physics is the GUT or the Planck scale we are at present bound to work with minimal theories that are valid up to high energy scales. This theory has in particular to be stable under the renormalization group evolution (RGE) which is an issue already in the SM. The measurement of the Higgs and the top-quark masses show that the SM is either in a marginally stable or in a metastable region of the parameter space $[25,26]$. However, as shown at two-loop level in 
$[14,27]$, these models not only provide a DM candidate but they also improve the stability of the SM and present a possibility to solve the baryon asymmetry problem.

In this article we explore possibilities of distinguishing the scalar and the vector DM (VDM) models. The minimal VDM requires an extra $U(1)$ gauge symmetry that is spontaneously broken by a vacuum expectation value (vev) of a complex scalar neutral field under the SM symmetries but charged under the extra $U(1)$. This model bears many similarities with a model of scalar DM (SDM) which is a component of an extra complex scalar field (that develops a vev) which is added to the SM. In both cases there are two scalar physical Higgs bosons $h_{1,2}$ that mix in the scalar mass matrix with a mixing angle $\alpha$. So the goal of this paper is to investigate if those two models could be distinguished. This is a very pragmatic task, both models are attractive candidates for simple DM theories, therefore it is worth knowing if there are observables which can distinguish them.

Using the SCANNERS program [28] we impose the most relevant bounds: theoretical, collider experiment bounds, precision electroweak physics, DM direct and indirect detection experiments, and DM relic density. The parameter space of each model is scanned with all the above constraints providing the regions of the parameter space where the models can indeed be distinguished. Whenever possible these results are presented in terms of physical observables that can be measured at the LHC. Finally we present a direct way to distinguishing the models by looking at the energy distribution in Higgs associated production, with the Higgs decaying to DM, at a future electron-positron collider.

The paper is organized as follows. In Sec. II we present the complex singlet extension of the SM, reviewing its main properties and setting notation. In Secs. II A and II B we discuss the scattering of scalar DM off nuclei and invisible SM-like Higgs boson decays, respectively. In Sec. III we set the review of most relevant aspects of the vector DM model. In Secs. III A and III B constraints from DM direct detection and invisible decays of SM-like Higgs boson are formulated, respectively. In Sec. IV we present a discussion of the possibility to distinguish the models at a future electron-positron collider. The results of the scan showing the allowed parameter space for each model are presented in Sec. VI. In the conclusions, Sec. VII, we summarize our findings. Technical details concerning Goldstone Boson couplings to Higgs bosons are left to the Appendices.

\section{SCALAR DARK MATTER}

Gauge singlet scalars as candidates for DM were first proposed in $[3,4]$ and then discussed by many authors. Even though the minimal model of scalar DM assumes merely an addition of a real scalar field odd under a $\mathbb{Z}_{2}$ symmetry, here we are going to consider a model that requires an extension by a complex scalar filed $S$. The motivation is to compare the VDM with a SDM that are in some sense similar. In order to stabilize a component of $S$ we require an invariance under DM charge conjugation $C: S \rightarrow S^{*}$, which guarantees stability of the imaginary part of $S, A \equiv \operatorname{Im} S / \sqrt{2}$. The real part, $\phi_{S} \equiv \operatorname{Re} S / \sqrt{2}$, is going to develop a real vacuum expectation value $\left\langle\phi_{S}\right\rangle=\langle S\rangle=v_{S} / \sqrt{2}$. ${ }^{1}$ Therefore $\phi_{S}$ will mix with the neutral component of the SM Higgs doublet $H$, in exactly the same manner as it happens for the VDM. In order to simplify the potential we impose in addition a $\mathbb{Z}_{2}$ symmetry $S \rightarrow-S$, which eliminates odd powers of $S$. Eventually the scalar potential reads:

$$
\begin{aligned}
V= & -\mu_{H}^{2}|H|^{2}+\lambda_{H}|H|^{4}-\mu_{S}^{2}|S|^{2}+\lambda_{S}|S|^{4}+\kappa|S|^{2}|H|^{2} \\
& +\mu^{2}\left(S^{2}+S^{* 2}\right)
\end{aligned}
$$

with $\mu^{2}$ real, as implied by the $C$ symmetry. Note that the $\mu^{2}$ term breaks the $U(1)$ explicitly, so the pseudo-Goldstone boson, $A$ is massive. In the limit of exact symmetry, $A$ would be just a genuine, massless Goldstone boson. Since the symmetry-breaking operator $\mu^{2}\left(S^{2}+S^{* 2}\right)$ is of dimension less that 4 , its presence does not jeopardize renormalizability even if noninvariant higher dimension operators were not introduced, see for instance [29]. Note that dimension 3 terms are disallowed by the $\mathbb{Z}_{2}$ 's and gauge symmetries. In other words, we can limit ourself to dimension $2 U(1)$ breaking terms preserving the renormalizability of the model. The freedom to introduce solely the soft breaking operators offers a very efficient and economical way to generate mass for the pseudoscalar $A$ without the necessity to introduce dimension 4 terms like $S^{4}$ or $|S|^{2} S^{2}$ and keeping the renormalizability of the model. It is also worth noticing that the $\mathbb{Z}_{2}$ symmetry $S \rightarrow-S$ is broken spontaneously by $v_{S}$ and therefore $\phi_{S}$, the real part of $S$, is not stable, making $A$ the only DM candidate.

The requirement of asymptotic positivity of the potential implies the following constraints that we impose in all further discussions:

$$
\lambda_{H}>0, \quad \lambda_{S}>0, \quad \kappa>-2 \sqrt{\lambda_{H} \lambda_{S}} .
$$

Hereafter the above conditions will be referred to as the positivity or stability conditions.

The scalar fields can be expanded around the corresponding generic vev's as follows

\footnotetext{
${ }^{1}$ This is a choice that fixes the freedom (phase rotation of the complex scalar) of choosing a weak basis that could be adopted to formulate the model. The model is defined by symmetries imposed in this particular basis in which the scalar vacuum expectation value is real.
} 


$$
\begin{aligned}
S & =\frac{1}{\sqrt{2}}\left(v_{S}+i v_{A}+\phi_{S}+i A\right), \\
H^{0} & =\frac{1}{\sqrt{2}}\left(v+\phi_{H}+i \sigma_{H}\right) \quad \text { where } H=\left(\begin{array}{c}
H^{+} \\
H^{0}
\end{array}\right),
\end{aligned}
$$

where we have temporarily allowed $\langle S\rangle$ to be complex.

Locations of extrema of the potential (2.1), corresponding values of the potential and corresponding curvatures in the basis $\left(\phi_{H}, \phi_{S}, A\right)$ are as follows

v1:

$$
\begin{aligned}
v^{2}= & \frac{4 \lambda_{S} \mu_{H}^{2}-2 \kappa\left(\mu_{S}^{2}-2 \mu^{2}\right)}{4 \lambda_{H} \lambda_{S}-\kappa^{2}}, \\
v_{S}^{2}= & \frac{4 \lambda_{H}\left(\mu_{S}^{2}-2 \mu^{2}\right)-2 \kappa \mu_{H}^{2}}{4 \lambda_{H} \lambda_{S}-\kappa^{2}}, \quad v_{A}^{2}=0 \\
V_{1}= & \frac{-1}{4 \lambda_{H} \lambda_{S}-\kappa^{2}}\left\{\lambda_{H}\left(\mu_{S}^{2}-2 \mu^{2}\right)^{2}\right. \\
& \left.+\mu_{H}^{2}\left[\lambda_{S} \mu_{H}^{2}-\kappa\left(\mu_{S}^{2}-2 \mu^{2}\right)\right]\right\} \\
\mathcal{M}^{2}= & \left(\begin{array}{ccc}
2 \lambda_{H} v^{2} & \kappa v v_{S} & 0 \\
\kappa v v_{S} & 2 \lambda_{S} v_{S}^{2} & 0 \\
0 & 0 & -4 \mu^{2}
\end{array}\right),
\end{aligned}
$$

v2:

$$
\begin{aligned}
v^{2}= & \frac{4 \lambda_{S} \mu_{H}^{2}-2 \kappa\left(\mu_{S}^{2}+2 \mu^{2}\right)}{4 \lambda_{H} \lambda_{S}-\kappa^{2}}, \quad v_{S}^{2}=0, \\
v_{A}^{2}= & \frac{4 \lambda_{H}\left(\mu_{S}^{2}+2 \mu^{2}\right)-2 \kappa \mu_{H}^{2}}{4 \lambda_{H} \lambda_{S}-\kappa^{2}}, \\
V_{2}= & \frac{-1}{4 \lambda_{H} \lambda_{S}-\kappa^{2}}\left\{\lambda_{H}\left(\mu_{S}^{2}+2 \mu^{2}\right)^{2}\right. \\
& \left.+\mu_{H}^{2}\left[\lambda_{S} \mu_{H}^{2}-\kappa\left(\mu_{S}^{2}+2 \mu^{2}\right)\right]\right\} \\
\mathcal{M}^{2}= & \left(\begin{array}{ccc}
2 \lambda_{H} v^{2} & 0 & \kappa v v_{S} \\
0 & 4 \mu^{2} & 0 \\
\kappa v v_{S} & 0 & 2 \lambda_{S} v_{S}^{2}
\end{array}\right),
\end{aligned}
$$

v3:

$$
\begin{gathered}
v^{2}=\frac{\mu_{H}^{2}}{\lambda_{H}}, \quad v_{S}^{2}=0, \quad v_{A}^{2}=0, \\
V_{3}=-\frac{\mu_{H}^{4}}{4 \lambda_{H}}
\end{gathered}
$$

$$
\mathcal{M}^{2}=\left(\begin{array}{ccc}
2 \mu_{H}^{2} & 0 & 0 \\
0 & 2 \mu^{2}+\frac{\kappa \mu_{H}^{2}}{2 \lambda_{H}}-\mu_{S}^{2} & 0 \\
0 & 0 & -2 \mu^{2}+\frac{\kappa \mu_{H}^{2}}{2 \lambda_{H}}-\mu_{S}^{2}
\end{array}\right) \text {, }
$$

v4:

$$
\begin{gathered}
v^{2}=0, \quad v_{S}^{2}=\frac{\mu_{S}^{2}-2 \mu^{2}}{\lambda_{S}}, \quad v_{A}^{2}=0, \\
V_{4}=-\frac{\left(\mu_{S}^{2}-2 \mu^{2}\right)^{2}}{4 \lambda_{S}}
\end{gathered}
$$

v5:

$$
\begin{gathered}
v^{2}=0, \quad v_{S}^{2}=0, \quad v_{A}^{2}=\frac{\mu_{S}^{2}+2 \mu^{2}}{\lambda_{S}}, \\
V_{5}=-\frac{\left(\mu_{S}^{2}+2 \mu^{2}\right)^{2}}{4 \lambda_{S}}
\end{gathered}
$$

Note that $v_{S} \neq 0$ and $v_{A} \neq 0$ may happen only if $\mu^{2}=0$. Since nonzero $\mu^{2}$ is essential to avoid the appearance of a Goldstone boson, we do not consider those points any more.

Forcing the vacuum $v 1$ to be the global minimum implies that we have to assume $\lambda_{H}>0,4 \lambda_{H} \lambda_{S}-\kappa^{2}>0$ and $\mu^{2}<0$. Then for consistency we enforce the conditions

$2 \lambda_{S} \mu_{H}^{2}>\kappa\left(\mu_{S}^{2}-2 \mu^{2}\right) \quad$ and $\quad 2 \lambda_{H}\left(\mu_{S}^{2}-2 \mu^{2}\right)>\kappa \mu_{H}^{2}$

It turns out that $V_{1}<V_{4}$ for any choice of parameters, while $V_{4}<V_{5}$ for $\mu^{2}<0$. From (2.17) one can find that the vacuum $v 3$ is never a minimum. Obviously, $v 2$ is not a minimum either for $\mu^{2}<0$. Therefore we conclude that for $\mu^{2}<0$ the vacuum $v l$ is the global minimum. Note that in this case $A$ is indeed a pseudo-Goldstone boson and its mass vanishes in the limit of exact global $U(1)$ as it was discussed and anticipated below (2.1). The presence of the $U(1)$ breaking term $\mu^{2}\left(S^{2}+S^{* 2}\right)$ implies a trivial shift of the $\mu_{S}^{2} \rightarrow \mu_{S}^{2}-2 \mu^{2}$ and an addition of the Goldstone boson mass $-4 \mu^{2}$. In fact, an equivalent $U(1)$ breaking would be to add just the Goldstone boson mass without the trivial shift by replacing $\mu^{2}\left(S^{2}+S^{* 2}\right)$ by $\mu^{2}\left(S-S^{*}\right)^{2}$.

Similar models have been considered in a more general context including a possibility of fast first order phase transition in $[7,20,30]$. In the VDM that we consider here, $A$ becomes a longitudinal component of the massive DM vector $X$, but it remains an independent degree of freedom. 
TABLE I. Vertices relevant for the calculation of annihilation cross section in the scalar DM model.

\begin{tabular}{l}
\hline \hline$i \frac{m_{i}^{2}}{v_{S}} R_{2 i}$ \\
\hdashline
\end{tabular}

There are two mass eigenstates, $h_{1}$ and $h_{2}$, in this model. The mass matrix (2.6) can be diagonalized by the orthogonal rotation matrix $R$ acting on the space spanned by the two $C P$-even scalars $\phi_{H}$ and $\phi_{S}$ :

$$
\left(\begin{array}{c}
\phi_{H} \\
\phi_{S}
\end{array}\right)=\left(\begin{array}{cc}
\cos \alpha & -\sin \alpha \\
\sin \alpha & \cos \alpha
\end{array}\right)\left(\begin{array}{l}
h_{1} \\
h_{2}
\end{array}\right) .
$$

We assume hereafter that $h_{1}$ is the $125 \mathrm{GeV}$ boson observed at the LHC.

Note that the third spin-zero state $A$ does not mix with the former ones as the $\mathbb{Z}_{2}$ dark symmetry remains unbroken by the real vev. We choose as independent parameters of the model the set: $v_{S}, \sin \alpha, m_{2}$, and $m_{A}$, while the parameters of the potential can be written as functions of this independent set and $v=246.22 \mathrm{GeV}$ and $m_{1}=125.09 \mathrm{GeV}$ as follows:

$$
\begin{aligned}
\kappa & =\frac{\sin 2 \alpha\left(m_{1}^{2}-m_{2}^{2}\right)}{2 v v_{S}}, \quad \lambda_{S}=\frac{\cos \alpha^{2} m_{2}^{2}+\sin \alpha^{2} m_{1}^{2}}{2 v_{S}^{2}}, \\
\lambda_{H} & =\frac{\cos \alpha^{2} m_{1}^{2}+\sin \alpha^{2} m_{2}^{2}}{2 v^{2}} .
\end{aligned}
$$

The vertices relevant for the calculation of annihilation cross section in the scalar DM model have been collected in Table I.

\section{A. Dark matter direct detections}

It is interesting to note that the DM direct detection signals are naturally suppressed in the scalar DM model. It turns out that in the limit of zero DM velocity the tree-level amplitude for DM-nucleon scattering vanishes. The most relevant interaction term in this context is the $A A h_{i}$ vertex. From the potential Eq. (2.1), one can easily derive the following DM triple-scalar couplings:

$$
\begin{aligned}
V & \supset \frac{A^{2}}{2}\left(2 \lambda_{S} v_{S} \phi_{S}+\kappa v \phi_{H}\right) \\
& =\frac{A^{2}}{2 v_{S}}\left(\sin \alpha m_{1}^{2} h_{1}+\cos \alpha m_{2}^{2} h_{2}\right),
\end{aligned}
$$

where we have used the relations Eqs. (2.18) and (2.19), and the corresponding Feynman rules are presented in Table I. With these interaction terms, we can write down the corresponding amplitude for the spin-independent DM nuclear recoils as follows:

$$
\begin{aligned}
i \mathcal{M} & =-i \frac{\sin 2 \alpha f_{N} m_{N}}{2 v v_{S}}\left(\frac{m_{1}^{2}}{q^{2}-m_{1}^{2}}-\frac{m_{2}^{2}}{q^{2}-m_{2}^{2}}\right) \bar{u}_{N}\left(p_{4}\right) u_{N}\left(p_{2}\right) \\
& \approx-i \frac{\sin 2 \alpha f_{N} m_{N}}{2 v v_{S}}\left(\frac{m_{1}^{2}-m_{2}^{2}}{m_{1}^{2} m_{2}^{2}}\right) q^{2} \bar{u}_{N}\left(p_{4}\right) u_{N}\left(p_{2}\right),
\end{aligned}
$$

where $q^{2}$ represents the DM momentum transfer when it scatters with nucleons, and $m_{N}$ and $f_{N} \approx 0.3$ denote the nucleon mass and its coupling to the SM Higgs. In the limit of zero momentum transfer, $q^{2} \rightarrow 0$, the above amplitude vanishes. This behavior is a consequence of the fact that the Goldstone-Higgs coupling is proportional the Higgs mass squared. In the Appendices we explain in a more general context when are the coupling of the form of (2.20), i.e., $\propto m_{i}^{2}$. It is interesting to note [31] that a similar cancellation also exists in a DM model with a vector gauge boson mediator which communicates with the SM sector only through kinetic mixings with the SM neutral gauge bosons. As shown in Ref. [31], it is even more remarkable that the cancellation in the vector mediator case does not demand to choose some specific soft breaking terms in the scalar potential, as in the present SDM.

It is shown in Ref. [32] that the leading-order DMnuclear recoil cross section arises at one-loop order, which is estimated as follows by assuming the one-loop functions to be of $\mathcal{O}(1)$

$$
\sigma_{A N} \approx \begin{cases}\frac{\sin ^{2} \alpha}{64 \pi^{5}} \frac{m_{N}^{4} f_{N}^{2}}{m_{1}^{4} v^{2}} \frac{m_{2}^{8}}{m_{A}^{2} v_{S}^{6}}, & m_{A} \geq m_{2} \\ \frac{\sin ^{2} \alpha}{64 \pi^{5}} \frac{m_{N}^{4} f_{N}^{2}}{m_{1}^{4} v^{2}} \frac{m_{2}^{4} m_{A}^{2}}{v_{S}^{6}}, & m_{A}<m_{2} .\end{cases}
$$

The above result is a conservative estimate of the upper limit for the one-loop $A$-nucleon scattering cross section. 
It turns out to be of $\mathcal{O}\left(10^{-49} \mathrm{~cm}^{2}\right)$ for $\sin \alpha=0.1, m_{2}=$ $300 \mathrm{GeV}$ and $m_{A} \sim 1 \mathrm{TeV}$, which is much lower than the current XENON1T limits of $\mathcal{O}\left(10^{-47} \mathrm{~cm}^{2}\right)$. Therefore, we expect that the DM direct searches will not impose any relevant constraints on the scalar DM model. In the following, we will use Eq. (2.22) to perform the scan which indeed confirms this expectation.

\section{B. Higgs-boson invisable decays: $h_{1} \rightarrow A A$}

One strong constraint for DM models comes from invisible decays of the SM-like Higgs boson, the corresponding branching ratio should be less than $24 \%$ [33]. In the present scalar DM model with $m_{A}<m_{1} / 2$, the SM Higgs boson decays invisibly into the stable pseudoscalar $\mathrm{DM} A, h_{1} \rightarrow A A$, with the decay width given by

$$
\Gamma\left(h_{1} \rightarrow A A\right)=\frac{1}{32 \pi} \frac{m_{1}^{2} \sin \alpha^{2}}{v_{S}^{2}} \sqrt{m_{1}^{2}-4 m_{A}^{2}} .
$$

\section{VECTOR DARK MATTER}

The model that we want to compare with the SDM is the popular vector DM (VDM) model [9-14] that is an extension of the SM by an additional $U(1)_{X}$ gauge symmetry and a complex scalar field $S$, whose vev generates a mass for this $U(1)$ 's vector field. The quantum numbers of the scalar field are

$S=(0, \mathbf{1}, \mathbf{1}, 1)$ under $U(1)_{Y} \times S U(2)_{L} \times S U(3)_{c} \times U(1)_{X}$.

None of the SM fields are charged under the extra gauge group. In order to ensure stability of the new vector boson a $\mathbb{Z}_{2}$ symmetry is assumed to forbid $U(1)$-kinetic mixing between $U(1)_{X}$ and $U(1)_{Y}$. The extra gauge boson $A_{\mu}$ and the scalar field $S$ transform under the $\mathbb{Z}_{2}$ as follows

$$
\begin{aligned}
A_{X}^{\mu} & \rightarrow-A_{X}^{\mu}, \quad S \rightarrow S^{*}, \text { where } S=\phi e^{i \sigma}, \\
\text { so } \phi & \rightarrow \phi, \quad \sigma \rightarrow-\sigma .
\end{aligned}
$$

All other fields are neutral under the $\mathbb{Z}_{2}$.

At leading order the vector bosons masses are given by:

$M_{W}=\frac{1}{2} g v, \quad M_{Z}=\frac{1}{2} \sqrt{g^{2}+g^{\prime 2}} v$ and $m_{X}=g_{X} v_{S}$,

where $g$ and $g^{\prime}$ are the $S U(2)$ and $U(1)$ gauge couplings, while $v$ and $v_{S}$ are $H$ and $S$ vev's: $(\langle H\rangle,\langle S\rangle)=\frac{1}{\sqrt{2}}\left(v, v_{S}\right)$. The scalar potential for this model is given by

$$
V=-\mu_{H}^{2}|H|^{2}+\lambda_{H}|H|^{4}-\mu_{S}^{2}|S|^{2}+\lambda_{S}|S|^{4}+\kappa|S|^{2}|H|^{2} .
$$

It will also be useful to define, for future reference, the parameter $\lambda_{\mathrm{SM}} \equiv m_{1}^{2} /\left(2 v^{2}\right)=0.13$, where $m_{1} \equiv 125.09 \mathrm{GeV}$.

The requirement of positivity for the potential implies the following constraints that we impose in all further discussions:

$$
\lambda_{H}>0, \quad \lambda_{S}>0, \quad \kappa>-2 \sqrt{\lambda_{H} \lambda_{S}} .
$$

It is easy to find the minimization conditions for the scalar fields (without losing generality one can assume $v$, $\left.v_{S}>0\right)$ :

$$
\begin{aligned}
& \left(2 \lambda_{H} v^{2}+\kappa v_{S}^{2}-2 \mu_{H}^{2}\right) v=0 \quad \text { and } \\
& \left(\kappa v^{2}+2 \lambda_{S} v_{S}^{2}-2 \mu_{S}^{2}\right) v_{S}=0
\end{aligned}
$$

If $\mu_{H, S}^{2}<0$ the global minimum at $(0,0)$ is the only extremum. For $\mu_{H, S}^{2}>0$ the point $(0,0)$ is a local maximum of the potential, in this case $\left(0, \frac{\mu_{S}}{\sqrt{\lambda_{S}}}\right)$ and $\left(\frac{\mu_{H}}{\sqrt{\lambda_{H}}}, 0\right)$ are global minima if $\kappa^{2}>4 \lambda_{H} \lambda_{S}$, otherwise they are saddle points and the global minima are determined by

$$
v^{2}=\frac{4 \lambda_{S} \mu_{H}^{2}-2 \kappa \mu_{S}^{2}}{4 \lambda_{H} \lambda_{S}-\kappa^{2}}, \quad v_{S}^{2}=\frac{4 \lambda_{H} \mu_{S}^{2}-2 \kappa \mu_{H}^{2}}{4 \lambda_{H} \lambda_{S}-\kappa^{2}} .
$$

For the VDM model only the latter case is relevant, since both vevs need to be non-zero to give rise to the masses of the SM fields and of the dark vector boson. Both scalar fields can be expanded around corresponding vev's as follows

$$
\begin{aligned}
S & =\frac{1}{\sqrt{2}}\left(v_{S}+\phi_{S}+i \sigma_{S}\right), \\
H^{0} & =\frac{1}{\sqrt{2}}\left(v+\phi_{H}+i \sigma_{H}\right) \quad \text { where } H=\left(\begin{array}{c}
H^{+} \\
H^{0}
\end{array}\right) .
\end{aligned}
$$

The mass squared matrix $\mathcal{M}^{2}$ for the fluctuations $\left(\phi_{H}, \phi_{S}\right)$ reads

$$
\mathcal{M}^{2}=\left(\begin{array}{cc}
2 \lambda_{H} v^{2} & \kappa v v_{S} \\
\kappa v v_{S} & 2 \lambda_{S} v_{x}^{2}
\end{array}\right),
$$

where the similarity to the mass matrix (2.6) in the SDM model is obvious. This mass matrix $\mathcal{M}^{2}$ can be diagonalized by the orthogonal rotation $R$ exactly as in (2.18) for the SDM. Note that here we adopt a convention such $h_{1}$ is the observed Higgs particle.

There are 5 real parameters in the potential: $\mu_{H}, \mu_{S}, \lambda_{H}$, $\lambda_{S}$, and $\kappa$. Adopting the minimization conditions (3.6) $\mu_{H}$, $\mu_{S}$ can be replaced by $v$ and $v_{S}$. Eventually there are 4 independent unknown parameters in the model and a convenient choice in this project is $v_{S}, \sin \alpha, m_{2}$, and $m_{X}$, which matches the choice made for the SDM model. 
TABLE II. The extra vertices relevant for the calculation of annihilation and scattering cross sections in the vector DM model.

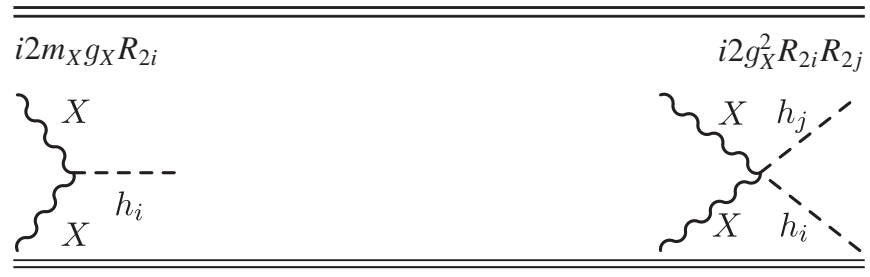

The parameters of the potential can be written as a function of the above set as:

$$
\begin{aligned}
\lambda_{H} & =\lambda_{\mathrm{SM}}+\sin ^{2} \alpha \frac{m_{2}^{2}-m_{1}^{2}}{2 v^{2}} \\
\kappa^{2} & =4\left(\lambda_{H}-\lambda_{\mathrm{SM}}\right) \frac{\lambda_{S} v_{S}^{2}-\lambda_{\mathrm{SM}} v^{2}}{v_{S}^{2}} \\
\lambda_{S} & =\frac{2 \kappa^{2}}{\sin ^{2} 2 \alpha} \frac{v^{2}}{m_{2}^{2}-m_{1}^{2}}\left(\frac{m_{2}^{2}}{m_{2}^{2}-m_{1}^{2}}-\sin ^{2} \alpha\right) .
\end{aligned}
$$

The extra vertices (besides those shown in Table I) needed for further calculations are collected in Table II.

\section{A. Dark matter direct detection}

The VDM model is constrained by the direct detection experiments. The spin-independent $X N$ scattering cross section is given by [34]

$$
\sigma_{X N}=\frac{\sin ^{2} 2 \alpha}{4 \pi} \frac{\left(m_{1}^{2}-m_{2}^{2}\right)^{2}}{m_{1}^{4} m_{2}^{4}} \frac{f_{N}^{2} \mu_{X N}^{2} m_{X}^{2} m_{N}^{2}}{v^{2} v_{S}^{2}}
$$

where $\mu_{X N} \equiv m_{X} m_{N} /\left(m_{X}+m_{N}\right)$ is the reduced mass in the DM-nucleon system. Note that compared with the pseudoscalar DM case in Eq. (2.22), it is clear that there is no suppression due to additional powers of relative DM velocity, thus we expect that the DM direct detection to results in a strong constraint to the present VDM model.

\section{B. Higg-boson invisable decays: $h_{1} \rightarrow X X$}

When the VDM mass is smaller than half of the SM-like Higgs boson $h_{1}, m_{X}<m_{1} / 2$, the Higgs invisible decay provides another constraint on the VDM scenario. In the present model, the width for invisible decays is provided by the process $h_{1} \rightarrow X X$ and can be expressed as follows [34]

$$
\Gamma\left(h_{1} \rightarrow X X\right)=\frac{g_{X}^{2} \sin ^{2} \alpha}{8 \pi} \sqrt{m_{1}^{2}-4 m_{X}^{2}} \frac{m_{X}^{2}}{m_{1}^{2}}\left[2+\frac{\left(m_{1}^{2}-2 m_{X}^{2}\right)^{2}}{4 m_{X}^{4}}\right] .
$$

\section{DISENTANGLING THE SCALAR AND VECTOR DM MODELS AT FUTURE LINEAR $e^{+} e^{-}$COLLIDERS}

The DM Higgs portal models can be tested by collider experiments [35-38]. The different DM scenarios were discussed using the effective operator approach $[39,40]$, simplified models [41-45] or other simple renormalizable models respecting unitarity and gauge-invariance [46-48]. An especially promising tool to probe the DM models discussed in this paper are future $e^{+} e^{-}$colliders [49]. In particular, they allow for the copious production of DM states associated with a $Z$ boson, what is referred to as Higgsstrahlung process or mono- $Z$ emision [31,50-55] (see the diagram in Fig. 1). We assume that the energy of the $Z$ boson can be reconstructed from data, therefore allowing for the determination of the missing energy, corresponding to the dark particles. The number of events observed for a given energy bin $\left(E_{Z}, E_{Z}+\Delta E_{Z}\right)$ allows to measure the value of the differential cross section, $\frac{d \sigma}{d E_{Z}}\left(E_{Z}\right)$, which is given by the following formula:

$$
\frac{d \sigma}{d E_{Z}}\left(E_{Z}\right)=f\left(s, E_{Z}\right) \cdot \frac{\left(\frac{\sin 2 \alpha}{v_{S}}\right)^{2} \cdot \sqrt{1-4 \frac{m_{\mathrm{DM}}^{2}}{Q^{2}}} \cdot\left(m_{1}^{2}-m_{2}^{2}\right)^{2} \cdot Q^{4}}{\left[\left(Q^{2}-m_{1}^{2}\right)^{2}+\left(m_{1} \Gamma_{1}\right)^{2}\right]\left[\left(Q^{2}-m_{2}^{2}\right)^{2}+\left(m_{2} \Gamma_{2}\right)^{2}\right]} \begin{cases}1 & (\mathrm{SDM}) \\ 1-4 \frac{m_{\mathrm{DM}}^{2}}{Q^{2}}+12\left(\frac{m_{\mathrm{DM}}^{2}}{Q^{2}}\right)^{2} & (\mathrm{VDM})\end{cases}
$$

where

$$
f\left(s, E_{Z}\right) \equiv \frac{\left(1-P_{+} P_{-}\right)\left(g_{v}^{2}+g_{a}^{2}\right)+2 g_{v} g_{a}\left(P_{+}-P_{-}\right)}{12 \cdot(2 \pi)^{3}} \sqrt{E_{Z}^{2}-m_{Z}^{2}}\left(2 m_{Z}^{2}+E_{Z}^{2}\right)\left(\frac{g^{2}}{\cos \theta_{W}^{2}} \frac{1}{s-m_{Z}^{2}}\right)^{2},
$$

$$
Q^{2}=Q^{2}\left(s, E_{Z}\right) \equiv s-2 E_{Z} \sqrt{s}+m_{Z}^{2}
$$

Here $g_{v}=\frac{1}{2}\left(1-4 \sin ^{2} \theta_{W}\right)$ and $g_{a}=\frac{1}{2}$ are the vector and axial couplings between electrons and the $Z$ boson, $g$ is the weak coupling constant, $m_{Z}$ is mass of the $Z$ boson and $\theta_{W}$ denotes the Weinberg angle. $P_{+}$and $P_{-}$ denote the polarization (defined as in [56]) of the positron and electron beam, respectively. Employing polarized beams can help to reduce the SM background (see Sec. IV D). The mass of the dark particle is denoted by $m_{\mathrm{DM}}$ (it is $m_{A}$ for the SDM and $m_{X}$ for the VDM) and $Q^{2}$ is the squared four-momentum of the decaying 


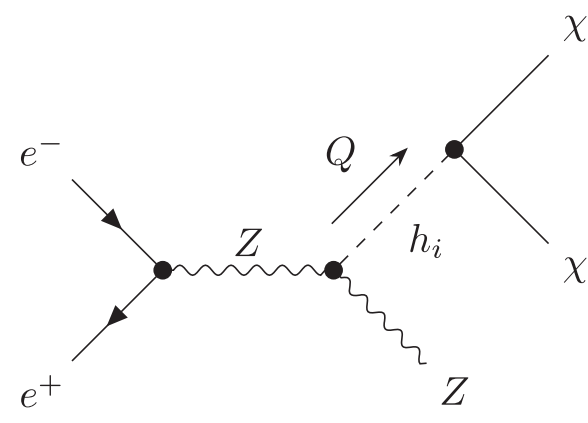

FIG. 1. Feynman diagram for considered channel of DM production. $\chi$ denotes the dark particle $(\chi=A, X)$.

Higgs particle. $\Gamma_{1}$ and $\Gamma_{2}$ are the total (including SM as well as dark channels) decay widths of $h_{1}$ and $h_{2}$, respectively, which must be calculated within each model as follows

$$
\begin{aligned}
\Gamma_{i}= & \Gamma_{i}^{\mathrm{SM}}+\frac{R_{2 i}^{2}}{32 \pi} \frac{m_{i}^{3}}{v_{S}^{2}} \sqrt{1-\frac{4 m_{\mathrm{DM}}^{2}}{m_{i}^{2}}} \\
& \cdot \begin{cases}1 & (\mathrm{SDM}) \\
1-4 \frac{m_{\mathrm{DM}}^{2}}{m_{i}^{2}}+12\left(\frac{m_{\mathrm{DM}}^{2}}{m_{i}^{2}}\right)^{2} & (\mathrm{VDM})\end{cases}
\end{aligned}
$$

where $\Gamma_{i}^{\mathrm{SM}}$ is the width of $h_{i}$ into SM final states. Note that the widths in (4.1) were dropped in the numerator as they are higher order terms in the perturbation expansion. Since $Q^{2} \geq 4 m_{\mathrm{DM}}^{2}$, the following important inequality holds

$$
\begin{aligned}
\max \left[\frac{3}{4}, f\left(x_{\min }\right)\right] & \geq 1-4 \frac{m_{\mathrm{DM}}^{2}}{Q^{2}}+12\left(\frac{m_{\mathrm{DM}}^{2}}{Q^{2}}\right)^{2} \\
& \geq \begin{cases}\frac{2}{3} & \text { if } x_{\min }<\frac{2}{3} \\
f\left(x_{\min }\right) & \text { if } \frac{2}{3}<x_{\min }<1\end{cases}
\end{aligned}
$$

where

$f(x) \equiv 1-x+\frac{3}{4} x^{2} \quad$ and $\quad x_{\min } \equiv\left(\frac{2 m_{\mathrm{DM}}}{\sqrt{s}-m_{Z}}\right)^{2}$.

Therefore from (4.1) we obtain the following solid prediction for the ratio of differential cross sections for SDM and VDM:

$$
\begin{aligned}
\left\{\max \left[\frac{3}{4}, f\left(x_{\min }\right)\right]\right\}^{-1} & \lesssim \frac{\frac{d \sigma_{\mathrm{SDM}}}{d E_{Z}}}{\frac{d \sigma_{\mathrm{VDM}}}{d E_{Z}}} \\
& \lesssim\left\{\begin{array}{ll}
\frac{3}{2} & \text { if } x_{\min }<\frac{2}{3} \\
{\left[f\left(x_{\min }\right)\right]^{-1}} & \text { if } \frac{2}{3}<x_{\min }<1
\end{array},\right.
\end{aligned}
$$

where it was assumed that the decay widths of $h_{1,2}$ are similar in both models. For cases adopted in this section $\max \left[3 / 4, f\left(x_{\min }\right)\right] \simeq 1$ therefore the left-hand side (lhs) of inequality (4.7) is very close to 1 while the right-hand side (rhs) is $3 / 2$. As a consequence of the above inequality, the total number of events predicted for the SDM model must be greater than for the VDM. The maximal deviation of the ratio of the distributions (4.1) from 1 corresponds to $Q^{2}=6 m_{\mathrm{DM}}^{2}$. Hence, it is easy to find that the distance $\delta$ between the energy $E_{Z}$ corresponding to the maximal deviation and the location of the $i$ th pole is

$$
\delta=\frac{m_{i}^{2}-6 m_{\mathrm{DM}}^{2}}{2 \sqrt{s}} .
$$

In turn, this means that the regions where the large ratio of the distributions (4.1) occur, are in the vicinity of a resonance (where the number of events is expected to be large). In particular, if $m_{i}^{2}=6 m_{\mathrm{DM}}^{2}$ the maximal deviation (50\%) appears exactly at the $i$ th pole.

An exemplary plot of $\frac{d \sigma}{d E_{Z}}$ is presented in Fig. 2. The maximal value of $E_{Z}$ for this process is

$$
E_{\max }=\frac{s-4 m_{\mathrm{DM}}^{2}+m_{Z}^{2}}{2 \sqrt{s}}
$$

what corresponds to $Q^{2}=4 m_{\mathrm{DM}}^{2}$. If $E_{Z}$ was higher, there would not be sufficient energy to produce the dark particles. Note that this threshold is clearly visible on the plot and we therefore assume that the mass of dark particles can be read from data.

The poles, $Q^{2}=m_{i}^{2}$, correspond to $h_{i}$ being on-shell. Therefore, the $i$ th pole is present if

$$
2 \cdot m_{\mathrm{DM}}<m_{i}<\sqrt{s}-m_{Z}
$$

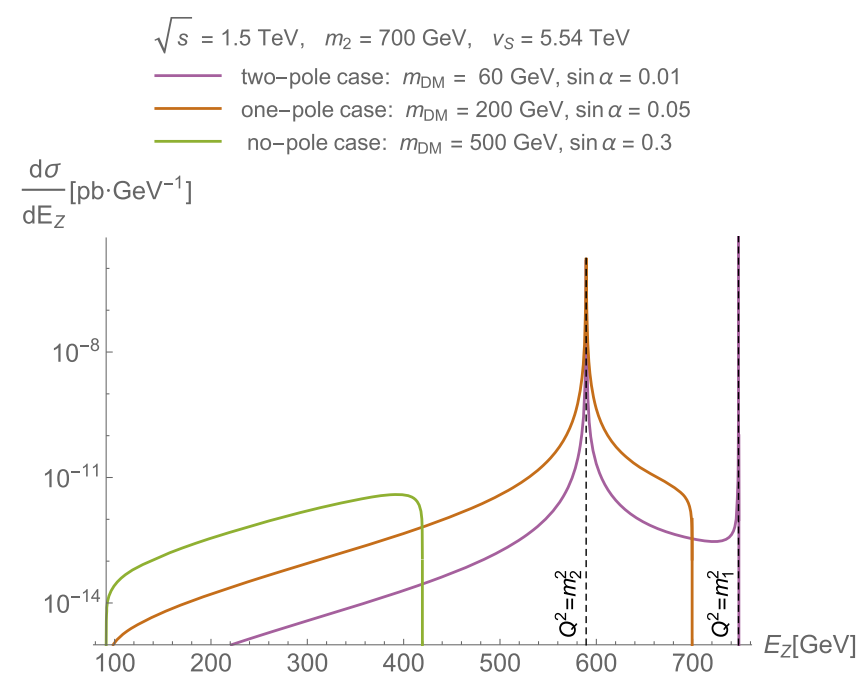

FIG. 2. An exemplary plot of $\frac{d \sigma}{d E_{Z}}$ function for the SDM model. Different curves correspond to different cases: for the purple one, $2 \cdot m_{\mathrm{DM}}<m_{1}, m_{2}$; for the brown $m_{1}<2 \cdot m_{\mathrm{DM}}<m_{2}$; and for the green $m_{1}, m_{2}<2 \cdot m_{\mathrm{DM}}$. 
In this case energy of $Z$ boson is

$$
E_{Z}\left(Q^{2}=m_{i}^{2}\right)=E_{i} \equiv \frac{s-m_{i}^{2}+m_{Z}^{2}}{2 \sqrt{s}},
$$

which in turn means that the mass of $h_{2}$ can be read from the position of the $h_{2}$ pole. If the $h_{2}$ pole is not present, $m_{2}$ has to be determined by an independent measurement.

Recently, two papers $[53,55]$ have discussed similar issues as the one described in this section. Their authors have considered the possibility to disentangle vector, scalar, and fermion DM at $e^{+} e^{-}$colliders. The vector model they adopted is the same as the one discussed here. However, for the scalar DM they used a minimal model with an extension by a real singlet, not by a complex one with softly broken global $U(1)$, which has been adopted here. Note that the scalar model considered here and the one adopted in $[53,55]$ are, in fact, very different. There, the coupling between DM and the mediator (the SM Higgs boson) is just given by the Higgs portal coupling (and the SM vev) and is independent of the mediator mass. In contrast, in the model discussed here, the DM is a pseudo-Goldstone boson of the spontaneously broken $U(1)$ symmetry. It is easy to see that in the limit of restored symmetry, i.e., when $\mu^{2} \rightarrow 0$, the $\mathrm{DM} A$ becomes a massless genuine Goldstone boson. As it is shown in the Appendices, in our case, with the $U(1)$ broken softly by the quadratic term $\mu^{2}\left(S^{2}+S^{* 2}\right)$, the coupling between the DM and the mediator $\left(h_{i}\right)$ is proportional to the mediator mass squared $m_{i}^{2} / v_{s}$. Note that in the VDM model the corresponding degree of freedom is a would-be Goldstone $G_{X}$ boson which becomes the longitudinal component of the massive vector DM $X$. Nevertheless, when one compares the ILC potential for those two versions of scalar DM vs VDM, it turns out that our conclusions are slightly less optimistic than those published in $[53,55]$.

In the following subsections we present a comparison of both DM models in a few typical cases for a $\sqrt{s}=1.5 \mathrm{TeV}$ collider.

\section{A. Two-pole case}

In this section we assume that both poles are present. As already mentioned $m_{2}$ and $m_{\mathrm{DM}}$ could be determined by the location of the $h_{2}$ resonance and by the endpoint of the distribution. We assume that $\sin \alpha$ and $v_{S}$ are known (deduced from some independent measurements), so that
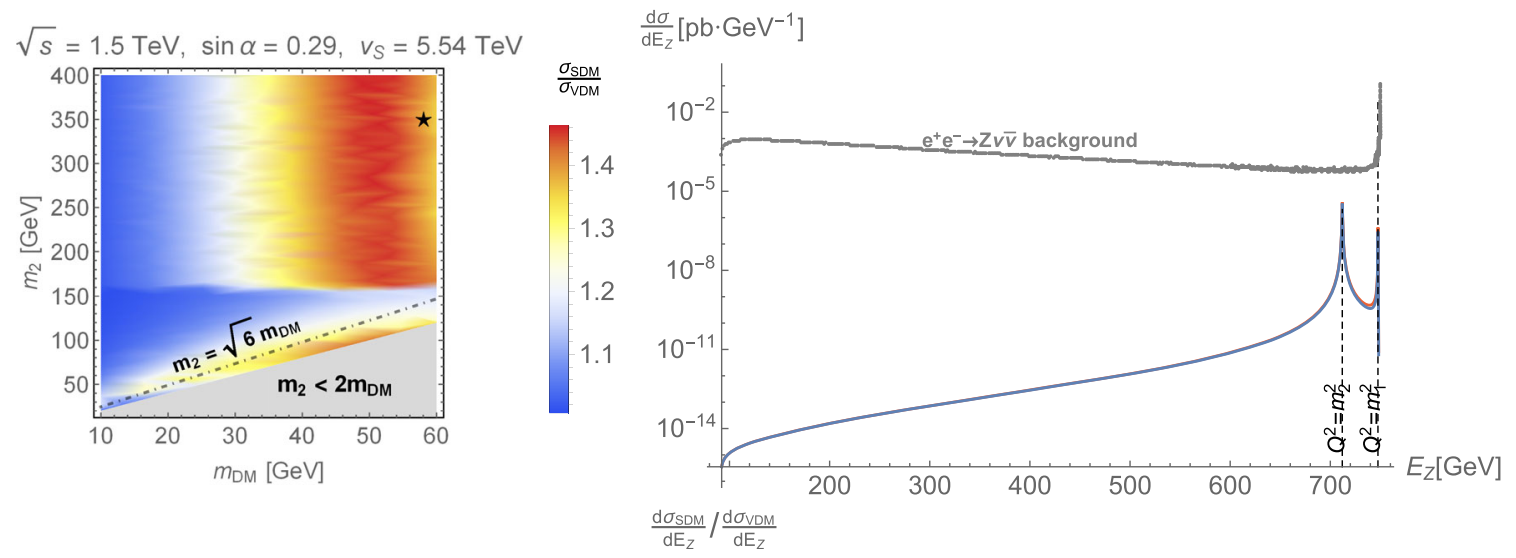

values in $\star$

$m_{2}=350 \mathrm{GeV}, \quad m_{\mathrm{DM}}=58 \mathrm{GeV}$

_ scalar DM model: $\sigma_{\text {tot }}=3.1 \times 10^{1} \mathrm{ab}$

$\Gamma_{1}=3.6 \times 10^{-3} \mathrm{GeV}, \mathrm{BR}_{h_{1} \rightarrow \mathrm{DM}}=0.6 \%$

$\Gamma_{2}=1.8 \mathrm{GeV}, \mathrm{BR}_{h_{2} \rightarrow \mathrm{DM}}=0.7 \%$

vector DM model: $\sigma_{\text {tot }}=2.2 \times 10^{1} \mathrm{ab}$

$\Gamma_{1}=3.6 \times 10^{-3} \mathrm{GeV}, \mathrm{BR}_{h_{1} \rightarrow \mathrm{DM}}=0.4 \%$

$\Gamma_{2}=1.8 \mathrm{GeV}, \mathrm{BR}_{h_{2} \rightarrow \mathrm{DM}}=0.6 \%$

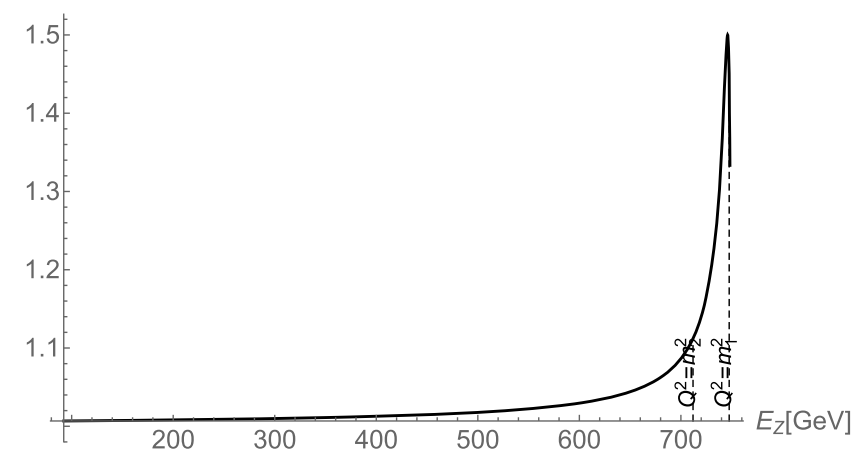

FIG. 3. Comparison of cross sections for the $e^{+} e^{-} \rightarrow Z h_{i}(\chi \chi)$ process $(\chi=A, X)$ for the SDM and for the VDM, in the two-pole case: $2 \cdot m_{\mathrm{DM}}<m_{1}, m_{2}$. The upper right panel shows $d \sigma / d E_{Z}$ for both models while the lower one shows the ratio of the distributions between the SDM and the VDM. The parameters chosen for the plot in the right panels are specified in the lower left corner and above the upper left panel. The chosen values for $\left(m_{\mathrm{DM}}, m_{2}\right)$ correspond to the point denoted by the star in the left upper panel. The colour bar shows the value of the ratio of total cross sections for $e^{+} e^{-} \rightarrow Z h_{i}(\chi \chi)$. The thick gray line on the upper right panel represents the SM background for our process (see Sec. IV D). Polarizations of the beams are $\left(P_{+}, P_{-}\right)=(-30 \%, 80 \%)$. 
we can compare the two models at the same points in the parameter space.

Figure 3 presents contours of the ratio of total cross sections $\sigma_{S D M} / \sigma_{V D M}$ in the $\left(m_{\mathrm{DM}}, m_{2}\right)$ space. The structure expected from (4.8) is visible, we observe the enhancement of the ratio for $m_{\mathrm{DM}} \simeq m_{1} / \sqrt{6} \simeq 51 \mathrm{GeV}$ and also for $m_{2} \simeq \sqrt{6} m_{\mathrm{DM}}$. In those regions $\sigma_{\mathrm{SDM}} / \sigma_{\mathrm{VDM}}$ reaches its maximal value $\sim 1.5$. The right panels show that, for the parameters chosen there, maximal enhancement of $d \sigma / d E_{Z}$ is observed near the resonance $Q^{2}=m_{1}^{2}$ and therefore a substantial value for the ratio of the total cross sections $(\sim 1.40)$ could be reached. The point in the parameter space adopted in the right panel satisfies all the experimental and theoretical constraints considered here. The region for which a two-pole scenario is not possible is marked in gray.

\section{B. One-pole case}

In this scenario we assume that $m_{1}<2 \cdot m_{\mathrm{DM}}<m_{2}$, therefore only one of the poles could be observed. Figure 4 shows the distribution functions and the ratio of the total cross sections in this case. We also show the contour plot of the ratio of total cross sections $\sigma_{\mathrm{SDM}} / \sigma_{\mathrm{VDM}}$ in the $\left(m_{\mathrm{DM}}, m_{2}\right)$ space. Since $m_{1}<2 \cdot m_{\mathrm{DM}}$ only the $h_{2}$ resonance appears. Again, for $m_{2} \simeq \sqrt{6} m_{\mathrm{DM}}$ the ratio of total cross sections is observed with maximal value close to 1.5 , i.e., maximal possible enhancement. There is only one enhancement band present in this case and the point in the parameter space adopted in the right panels satisfy all the experimental and theoretical constraints considered here. The point has been chosen such that the maximal ratio of the differential cross sections is observed near the resonance, so that the ratio of the total cross sections can reach $\sim 1.45$.

\section{No-pole case}

In this case no pole is present since both Higgs particles are lighter than $2 \cdot m_{\mathrm{DM}}$. Again we adopt a similar strategy to illustrate this case. The difference is that since there is no pole present the mechanism to amplify the ratio of $\sigma_{\mathrm{SDM}} / \sigma_{\mathrm{VDM}}$ does not work. As a result, the contour plots for the ratio of the total cross sections show only very mild enhancement this time. These results are shown in Fig. 5.

\section{Expected statistical error and SM background}

The expected statistical error for measurements of the total cross section is equal to

$$
\Delta \sigma=\sqrt{\frac{\sigma_{\mathrm{sig}}+\sigma_{\mathrm{bg}}}{\eta \int \mathscr{L} d t}},
$$

where $\eta$ stands for the efficiency of detectors, $\int \mathscr{L} d t$ is the luminosity of the collider integrated over the whole data

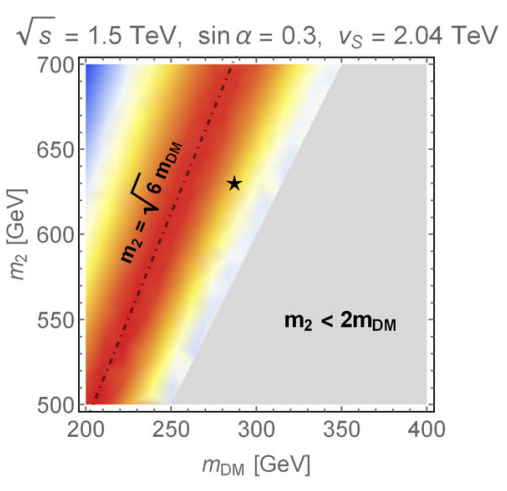

values in $\star$

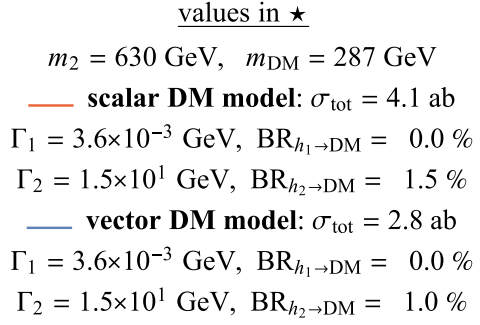

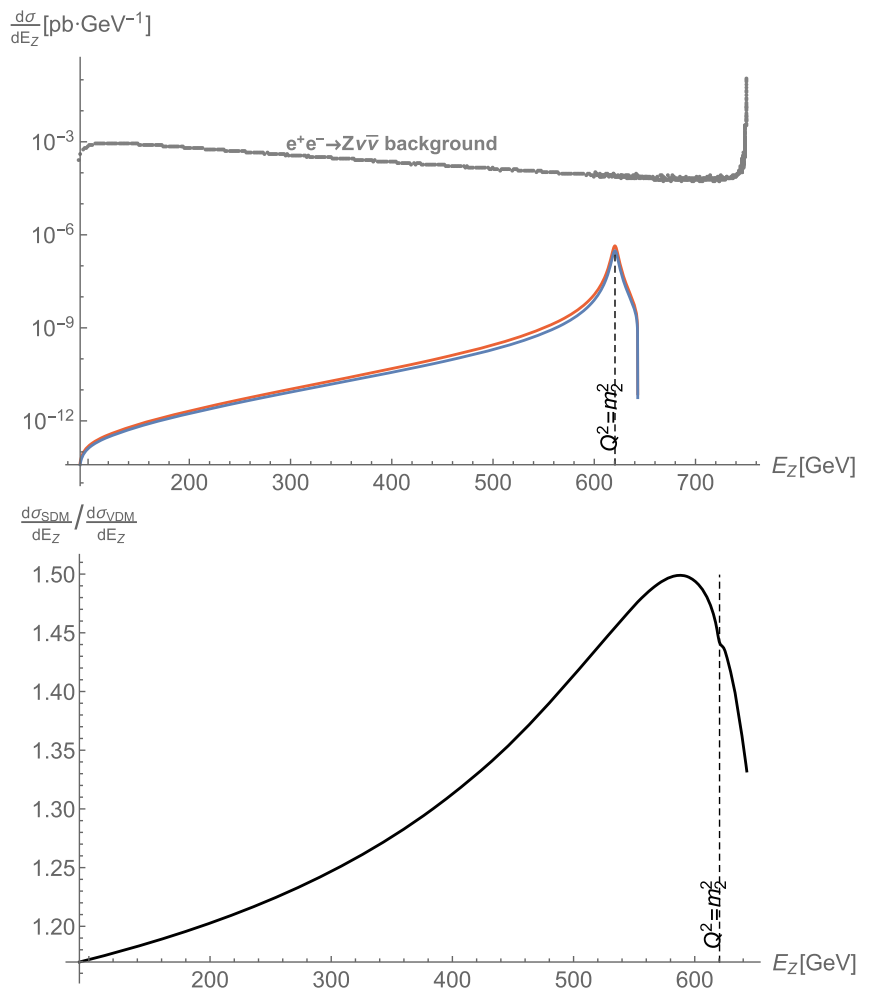

FIG. 4. As in Fig. 3, however for the one-pole case, i.e., for $m_{1}<2 \cdot m_{\mathrm{DM}}<m_{2}$. 

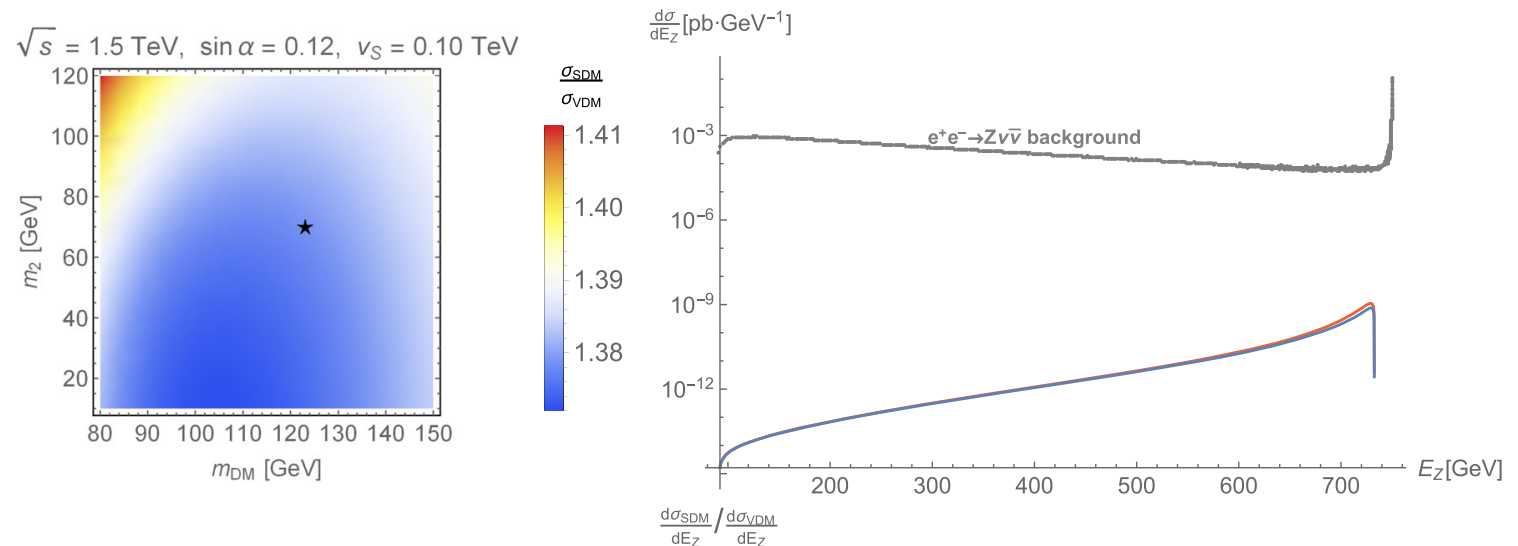

values in $\star$

$m_{2}=70 \mathrm{GeV}, \quad m_{\mathrm{DM}}=123 \mathrm{GeV}$

$$
\begin{gathered}
\text { scalar DM model: } \sigma_{\text {tot }}=3.1 \times 10^{-2} \mathrm{ab} \\
\Gamma_{1}=3.9 \times 10^{-3} \mathrm{GeV}, \mathrm{BR}_{h_{1} \rightarrow \mathrm{DM}}=0.0 \% \\
\Gamma_{2}=2.5 \times 10^{-5} \mathrm{GeV}, \mathrm{BR}_{h_{2} \rightarrow \mathrm{DM}}=0.0 \% \\
\text { vector DM model: } \sigma_{\text {tot }}=2.2 \times 10^{-2} \mathrm{ab} \\
\Gamma_{1}=3.9 \times 10^{-3} \mathrm{GeV}, \mathrm{BR}_{h_{1} \rightarrow \mathrm{DM}}=0.0 \% \\
\Gamma_{2}=2.5 \times 10^{-5} \mathrm{GeV}, \mathrm{BR}_{h_{2} \rightarrow \mathrm{DM}}=0.0 \%
\end{gathered}
$$

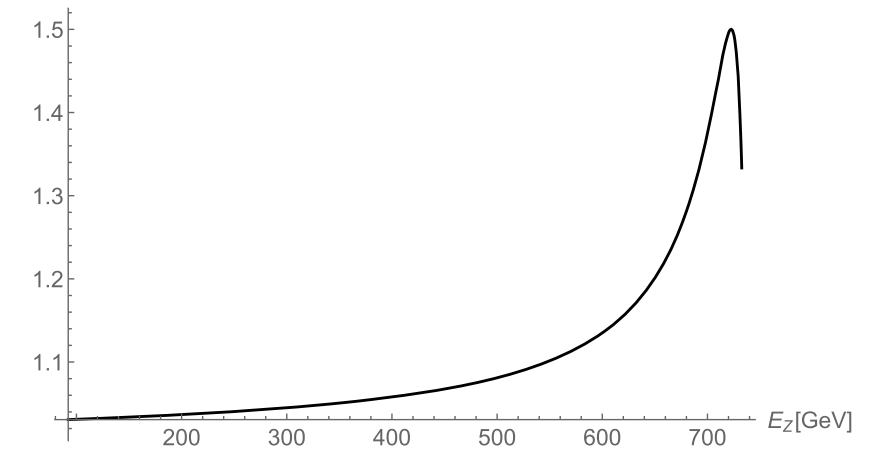

FIG. 5. As in Fig. 3, however for the no-pole case: $m_{1}, m_{2}<2 \cdot m_{\mathrm{DM}}$.

collection period for a given $\sqrt{s}, \sigma_{\text {sig }}$ is the total cross section for the signal and $\sigma_{\mathrm{bg}}$ is the background cross section. Following [57,58], we assume that

$$
\begin{aligned}
& \eta \approx 1,\left.\quad \int \mathscr{L} d t\right|_{\mathrm{CLIC}, \sqrt{s}=1.5 \mathrm{TeV}} \approx 1500 \mathrm{fb}^{-1}, \\
& \left.\int \mathscr{L} d t\right|_{\mathrm{CEPC}, \sqrt{s}=240 \mathrm{GeV}} \approx 3000 \mathrm{fb}^{-1} .
\end{aligned}
$$

In order to decide whether the two models will be experimentally distinguishable, we can compare the difference between the total cross sections for SDM and VDM to its uncertainty

$$
\begin{aligned}
\Delta\left(\sigma_{\mathrm{SDM}}-\sigma_{\mathrm{VDM}}\right) & =\sqrt{\left(\Delta \sigma_{\mathrm{SDM}}\right)^{2}+\left(\Delta \sigma_{\mathrm{VDM}}\right)^{2}} \\
& =\sqrt{\frac{\left(\sigma_{\mathrm{SDM}}+\sigma_{\mathrm{bg}}\right)+\left(\sigma_{\mathrm{VDM}}+\sigma_{\mathrm{bg}}\right)}{\eta \int \mathscr{L} d t}} .
\end{aligned}
$$

Production of dark particles can be mimicked by processes that produce missing energy in the form of SM neutrinos that escape detection. The simplest and most relevant example of such a process, with a similar experimental signature, that is the irreducible background, is $e^{+} e^{-} \rightarrow$ $Z Z^{*} \rightarrow Z \nu \bar{\nu}$, where $\nu=\nu_{e}, \nu_{\mu}, \nu_{\tau}$ (see the exemplary
Feynman diagrams in Fig. 6). The background could be reduced to some extent by employing polarized $e^{+} e^{-}$ beams. According to the current predictions for the ILC [59], polarization of the beams should be possible at the level of $80 \%$ for electrons and $30 \%$ for positrons. The expected background, presented in Figs. 3-5, is calculated under that assumption using CALCHEP [60]. The background is a serious obstacle in the determination of parameters for DM particles. One can see that among our examples only in the two-pole case in the vicinity of the SM-like resonance $h_{1}$, the signal is comparable to the background. In order to increase the signal-to-background ratio, one should be collecting only events in a vicinity of a pole for one of the Higgs bosons, i.e., only events with $Z$ boson energy within a certain bin around $E_{Z}=E_{i}(\sqrt{s}) \equiv$ $\left(s-m_{i}^{2}+m_{Z}^{2}\right) /(2 \sqrt{s})$. It turns out that for $\sqrt{s}=1.5 \mathrm{TeV}$, $m_{\mathrm{DM}}=44.5 \mathrm{GeV}, \quad m_{2}=102 \mathrm{GeV}, \quad v_{S}=5 \mathrm{TeV}$, and $\sin \alpha=0.31$ the separation between the cross sections for SDM and VDM at the level of $1 \sigma$ could be obtained for a bin around $E_{Z}=E_{1}(1.5 \mathrm{TeV})$ with the width $\sim 4.5 \mathrm{GeV}$. To estimate the minimal value of experimental uncertainty of $E_{Z}$ we assumed that energy of the $Z$ boson is reconstructed from energy of jets that are produced, since the branching ratio of $Z$ into hadrons is almost $70 \%$ [61]. The jet energy can be measured in calorimeters with resolution $~ 3 \%$ $[58,62]$. Hence, the minimal size expected for the resolution of the $Z$ energy near the $h_{1}$ pole at, for instance, CLIC is $\sim 3 \% \times\left. E_{Z}\right|_{E_{Z}=E_{1}(1.5 \mathrm{TeV})}=22.4 \mathrm{GeV}$, and so it seems 

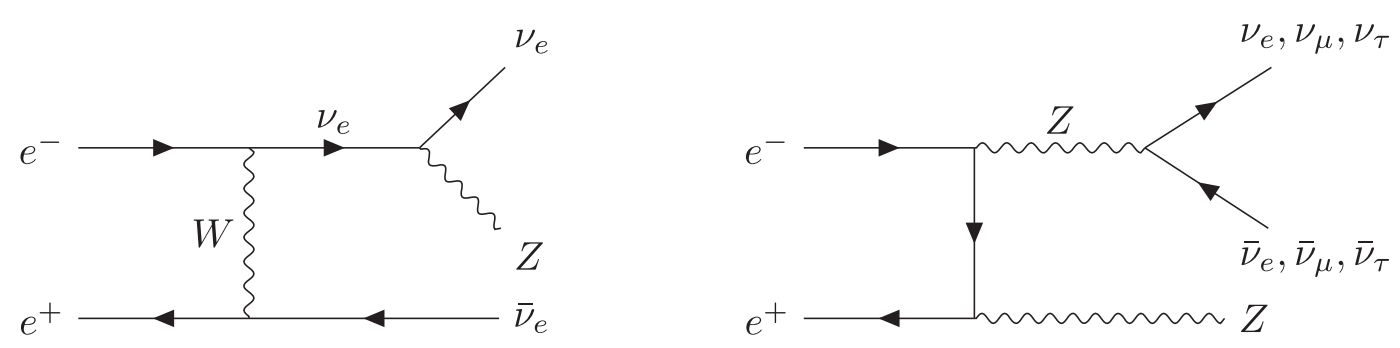

FIG. 6. Exemplary diagrams of the standard model background processes. Neutrinos contribute to missing energy and can therefore mimic dark particles. The background cross section could be reduced by polarizing the initial $e^{+}$and $e^{-}$beams.

extremely hard to disentangle the two scenarios for the adopted parameters at this collider. On the other hand, for $\sqrt{s}=240 \mathrm{GeV}$ expected for the CEPC and the same parameters, for the minimal bin size $\sim 3 \% \times$ $\left.E_{Z}\right|_{E_{Z}=E_{1}(240 \mathrm{GeV})}=3.1 \mathrm{GeV}$ the separation between the two cross sections is at the level of $12 \sigma$. Therefore it is fair to conclude that there exist regions of parameters, where the two scenarios might be disentangled at future $e^{+} e^{-}$colliders in resonance regions. However, without a detailed error and background analysis that takes into account all experimental details it is impossible to draw any solid final conclusions.

\section{NUMERICAL SIMULATION}

The two models described in the previous sections were implemented in the SCANNERS [28,63] code as model classes. The code takes as input any scalar potential that is a polynomial in the fields of order up to four and by considering the VEVs, mixing angle and physical masses as independent parameters, turns the problem of deriving the original potential parameters into a set of linear equations, with a very significant increase in speed of the scanning process (see [28] for details). In the most general cases, the drawback of this method is that a given point is only verified to be a global minimum at the end of the procedure. However, because it is easy to obtain closed conditions for the global minimum for the particular models under study, this problem is avoided. The code is equipped with a set of tools which allow to automatize the parameter scans and also with generic modules that allow to test local vacuum stability and library interfaces to the constraints implemented for each model. SCANNERS is also interfaced with other high energy tools that simplify the implementation of the constraints that will be described shortly.

TABLE III. Independent parameters' range for both models.

\begin{tabular}{lc}
\hline \hline Parameter & Range \\
\hline SM-Higgs $-m_{1}$ & $125.09 \mathrm{GeV}$ \\
Second Higgs- $m_{2}$ & {$[1,1000] \mathrm{GeV}$} \\
DM- $m_{\mathrm{DM}}$ & {$[1,1000] \mathrm{GeV}$} \\
Singlet VEV $-v_{s}$ & {$\left[1,10^{7}\right] \mathrm{GeV}$} \\
Mixing angle- $\alpha$ & {$\left[-\frac{\pi}{4}, \frac{\pi}{4}\right]$} \\
\hline \hline
\end{tabular}

The ranges for the independent parameters are listed in Table III. The ranges are the same for both models under study.

The points generated using SCANNERS have to be in agreement with the most relevant experimental and theoretical constraints. The discovered Higgs boson mass is taken to be $m_{h}=125.09 \mathrm{GeV}$ from the ATLAS/CMS combination [64]. In these models the Higgs couplings to remaining SM particles are all modified by the same factor. Therefore, the bound on the signal strength [64] is used to constrain this parameter. The vacuum expectation value of the Higgs doublet is fixed by the W-mass. The points generated have to comply with the following theoretical constraints: (i) the potential has to be bounded from below; (ii) the vacuum is chosen so that the minimum is the global one and (iii) perturbative unitarity holds. The first two constraints are implemented in the code while perturbative unitarity is imposed trough an internal numerical procedure that includes all possible two to two processes and that is available in SCANNERS for a generic model. In these models new contributions to the radiative corrections of the massive gauge-boson self-energies, $\Pi_{W W}\left(q^{2}\right)$ and $\Pi_{Z Z}\left(q^{2}\right)$ appear via the mixing between the neutral components of the doublet and the singlet. We use the variables $\mathrm{S}, \mathrm{T}, \mathrm{U}$ [65] (expressions available in [66]) to guaranty that the models are in agreement with the electroweak precision measurements at the $2 \sigma$ level.

The phenomenological constraints are imposed either via libraries in the code or with interfaces with other high energy codes. The collider bounds from LEP, Tevatron, and the LHC are all encoded in HiggsBounds [67]. The program can be used to ensure agreement at $95 \%$ confidence level exclusion limits for all available searches for non-standard Higgs bosons. The Higgs decay widths, including the state-of-the art higher order QCD corrections were calculated with SHDECAY [63]. ${ }^{2}$ SHDECAY is based on the implementation of the models in HDECAY $[68,69]$. In our calculations all electroweak radiative corrections are turned off for consistency. A detailed description of the program can be found in Appendix A of [63].

\footnotetext{
${ }^{2}$ The program sHDECAY can be downloaded from the url: http://www.itp.kit.edu/maggie/sHDECAY.
} 
Up until run 2 of the LHC only LEP had constraints on resonances below $100 \mathrm{GeV}$ after the discovery of the Higgs boson. New analyses using data from the run 2 of the LHC, at $13 \mathrm{TeV}$, constrain now the production and decay rates of heavy resonances into gauge bosons [70-72] and into SM-like Higgs pairs [73,74]. These bounds are included in our analysis. The most stringent of the bounds is by far the one from [70]. However, as we will show, even if a substantial number of points are excluded by this analysis, the values of $\sin \alpha$ allowed by the very strong constraints on the $125 \mathrm{GeV}$ Higgs couplings to SM particles (modified by the common factor $\cos \alpha$ that affects all couplings in the same way) are roughly in the same region.

For the DM phenomenology, we consider the constraints from the cosmological DM relic abundance, collider searches, DM direct and indirect detections. The DM relic abundance for each model is calculated with the MicrOMEGAs code [75], which is compared with the current experimental result $\left(\Omega h^{2}\right)_{\mathrm{DM}}^{\mathrm{obs}}=0.1186 \pm 0.002$ from the Planck Collaboration [76]. Note that here we do not restrict the DM relic abundance to be exactly at the experimental value. Rather, we only require the model predicted value be equal to or smaller than the observed one. This way, we can consider both the dominant and subdominant DM cases simultaneously, for which we define the following DM fraction

$$
f_{A, X}=\frac{\left(\Omega h^{2}\right)_{A, X}}{\left(\Omega h^{2}\right)_{\mathrm{DM}}^{\mathrm{obs}}},
$$

where $\left(\Omega h^{2}\right)_{A, X}$ denote the calculated DM relic abundance for either the pseudoscalar DM $A$ or the VDM $X$.

The Higgs portal couplings can induce spin-independent DM-nucleon recoils for both scalar and vector DM models and the corresponding expressions have already been presented in Eqs. (2.22) and (3.13). Currently, the LUX [77], PandaX-II [78], and XENON1T [79,80] experiments give the most stringent upper bounds for the DM nucleon scattering. In our work, we apply the latest XENON1T upper bounds [80] for DM mass greater than $6 \mathrm{GeV}$, while for lighter DM particles, the combined limits from CRESST-II [81] and CDMSlite [82] are used. Note that these experimental DM-nucleon scattering upper limits were derived by assuming that the DM candidate comprises all of DM abundance. Therefore, the proper quantity to be directly compared with experimental limits should be the effective DM-nucleon cross section defined by $\sigma_{A N, X N}^{\text {eff }} \equiv f_{A, X} \sigma_{A N, X N}$.

The DM indirect detection experiments can also impose strong constraints on the DM properties. In the models considered in the present work, the annihilation of DM into visible states may manifest itself in: the temperature anisotropies of CMB radiation, the $\gamma$-ray signals in the spheroidal dwarf galaxies or as the $e^{ \pm}$excesses in the Milky Way what can be probed and constrained by the observations of Planck [76], Fermi-LAT [83], and AMS-02 [84,85], respectively. According to Ref. [86], it is shown that for the DM mass range of interest, the FermiLAT upper bound on the DM annihilations from dwarfs is the most stringent. Note that both for the scalar and vector DM models, most of DM annihilations through the Higgs portal goes into $\mathrm{ZZ}, W^{+} W^{-}, b \bar{b}$ and light quark pairs. According to Ref. [83], all of these final states give nearly the same upper limits on the DM annihilation cross sections. Thus, we use the Fermi-LAT bound from Ref. [83] on $b \bar{b}$ when $m_{A, X} \geq m_{b}$, and that on light quarks for $m_{A, X}<m_{b}$. Also, similar to the DM direct detections, the comparison with the data requires the use of the effective DM annihilation cross sections defined by $\sigma_{A A, X X}^{\text {eff }}=f_{A, X}^{2} \sigma_{A A, X X}$, which are computed with the MicrOMEGAs code [75] automatically.

Collider searches can provide information on DM particles through the SM-like Higgs $h_{1}$ invisible decay, with the corresponding decay width given in Eqs. (2.23) and (3.14) for both DM models. The predicted Higgs invisible decay branching ratios should be compared with the LHC bound on this channel $\operatorname{Br}\left(h_{1} \rightarrow\right.$ inv $)=0.24$ [33].

\section{RESULTS}

In this section we compare the available parameter space for the two models after applying all the constraints described in Sec. V. Again we note that the models have the same number of independent parameters. From the phenomenological point of view, the experimental measured quantities are the same, the second Higgs mass, the DM mass, the mixing angle $\alpha$ and the singlet VEV. It is clear that the LHC cannot prove the existence of DM if it is not confirmed by direct detection experiments. It is also true that the existence of a second neutral Higgs is predicted in most of the simplest extensions of the scalar sector. However, if a new scalar is discovered while a hint for DM appears in the form of say, mono-X events, it may be possible to exclude some DM models if the events are in a region of the parameter space already excluded. In the remainder of this section the color code in the figures is the following: red is for scalar DM and blue for vector DM, and in both cases relic density is not saturated, meaning that extra DM candidates are needed; on top of those points we present the points that are within $5 \sigma$ of the central value of the relic abundance value, in pink for the scalar case and in purple for the vector case. The colors are superimposed in the following order: red, blue, pink, and then purple (so for instance a red dot may be hidden behind a blue dot).

We start with Fig. 7 where we present the branching ratio of $h_{2} \rightarrow h_{1} h_{1}$ as a function of $m_{2}$ for the scalar model and for the vector model. Clearly, there is no significant difference between the two models. Values for the branching ratio reach a maximum of $70 \%$ just after the channel opens and then reduces to maximum values of about $40 \%$. However, 


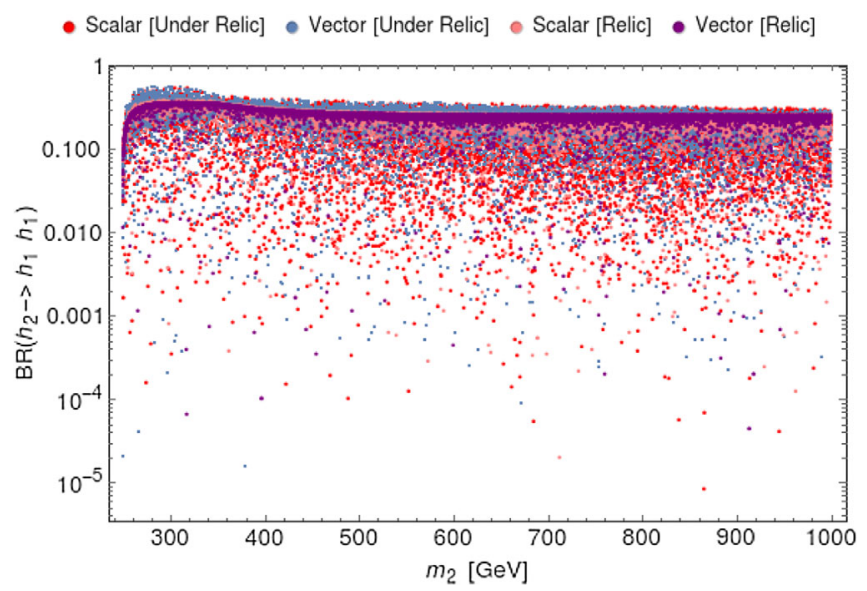

FIG. 7. Branching ratio of $h_{2} \rightarrow h_{1} h_{1}$ as a function of $m_{2}$ for the scalar model and for the vector model (color code in the legend).

if relic density is saturated the branching ratio is mostly below $40 \%$ and again indistinguishable for the two models.

In Fig. 8 we plot the branching ratio of the SM-like Higgs (a) and of the second Higgs (b) into DM particles

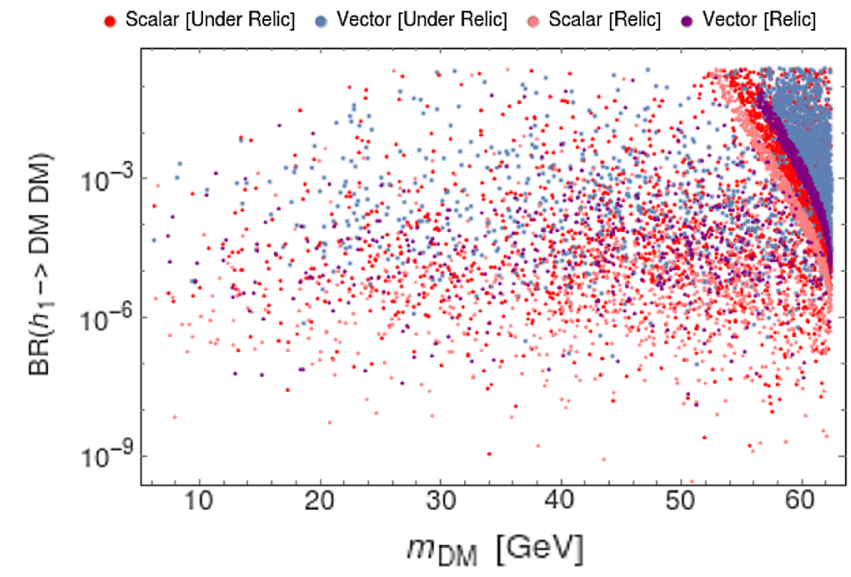

(a) SM-like Higgs. as a function of the DM mass. Once more no significant deviations can be seen between the models and in this case there is no difference from the saturated to the nonsaturated scenario.

In the left panel of Fig. 9 we plot $m_{\mathrm{DM}} / v_{S}$, a quantity that reduces to the gauge coupling constant in the VDM model:

$$
\frac{m_{\mathrm{DM}}}{v_{S}}= \begin{cases}g_{X} & \text { for } \mathrm{VDM} \\ \frac{m_{A}}{v_{S}} & \text { for SDM }\end{cases}
$$

Roughly the same region is populated by both models. Note that points with suppressed $m_{\mathrm{DM}} / v_{S}$ in the range between $10^{-4}$ and $10^{-2}$ for $m_{\mathrm{DM}} \lesssim 500 \mathrm{GeV}$ correspond to $h_{2}$ resonances. In our scan $m_{2}$ varies between 1 and $1000 \mathrm{GeV}$, therefore the resonances $\left(m_{\mathrm{DM}} \sim m_{2} / 2\right)$ are distributed nearly uniformly for $1 \mathrm{GeV} \lesssim m_{\mathrm{DM}} \lesssim 500 \mathrm{GeV}$. For those points the requirement of proper DM abundance imply suppression of the coupling between DM and the resonance, so that $m_{\mathrm{DM}} / v_{S}$ must be small.

FIG. 8. Branching ratio of the SM-like Higgs (a) and of the second Higgs (b) into DM particles as a function of the DM mass.
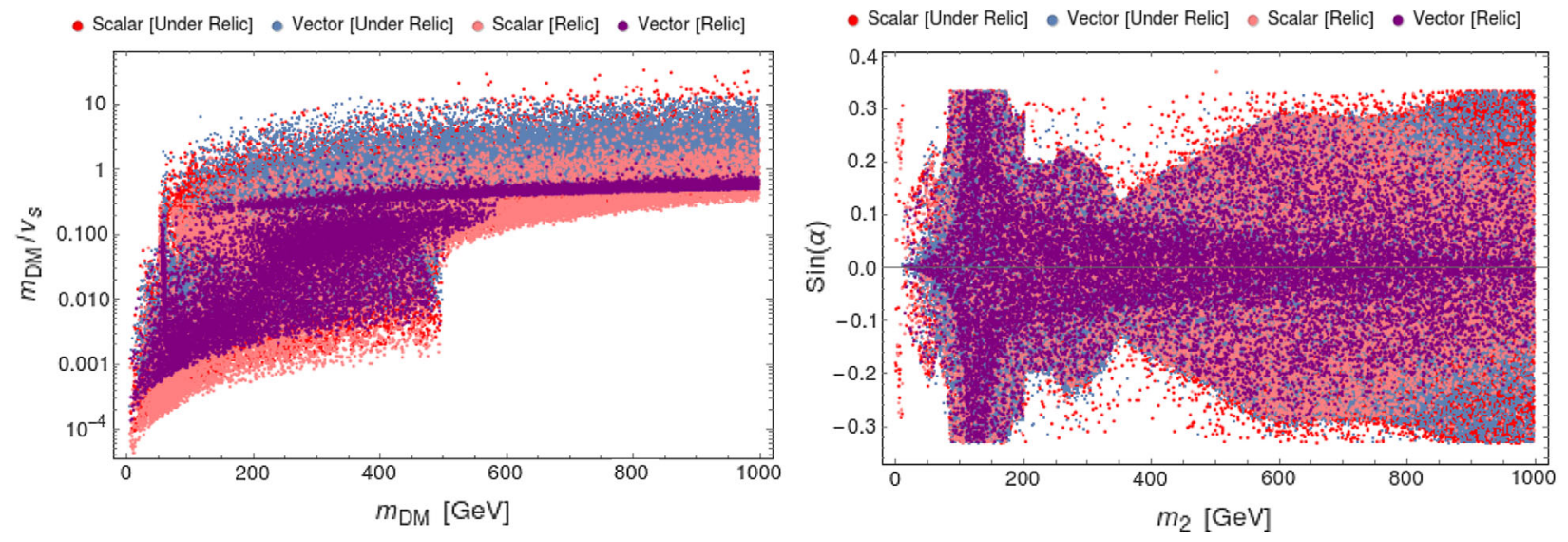

FIG. 9. Left: $m_{\mathrm{DM}} / v_{S}$ as a function of the DM mass; right: $\sin \alpha$ as a function of $m_{2}$. 
In the right panel of Fig. 9 we show $\sin \alpha$ as a function of the second Higgs mass. The allowed band between about -0.34 and 0.34 for $m_{2}$ above roughly $m_{1} / 2$ is a hard ( $m_{2}$-independent) bound on $\sin \alpha$ that comes from the combined signal strength measurements of the production and decay of the SM-like Higgs, $h_{1}$. This bound is weaker than in case of the real singlet model with no DM candidate. The reason is that both $\operatorname{BR}\left(h_{1} \rightarrow V V\right)$ and $\operatorname{BR}\left(h_{1} \rightarrow f \bar{f}\right)$ might be reduced if $m_{\mathrm{DM}}<m_{1} / 2$. The "pattern" of densely populated points is visible in the right panel of Fig. 9. Those points are originating from constraints imposed by searches for heavy scalar resonances, i.e., $h_{2}$ in our case. The pattern originates mainly from the search for $p p(g g) \rightarrow h_{2} \rightarrow Z Z$. Let us focus on this final state. The $h_{2}$ production cross section is the same as for the SM multiplied by $\sin ^{2} \alpha$. Therefore, the shape of the pattern reflects mostly the behavior of the SM cross section $\sigma\left(h_{\mathrm{SM}}\right)$ as a function of $m_{\mathrm{SM}}$ that is here replaced by $m_{2}$. That is the reason why the exclusion is maximal close to the $t \bar{t}$ threshold, where the Higgs production cross section via gluon fusion has a local maximum. Notice also the presence of less densely populated regions outside of the pattern with relatively large values of $\sin \alpha$. In order to understand its appearance, one should note that the total width of the second Higgs has an extra contribution $\Gamma\left(h_{2} \rightarrow\right.$ DMDM $)$. Therefore, in contrast to what happens in the singlet extension with no dark matter candidate, here the $\operatorname{BR}\left(h_{2} \rightarrow\right.$ ZZ) might be suppressed implying larger allowed values of $\sin \alpha$ located outside of the pattern. To illustrate this point we plot in Fig. $10 \mathrm{BR}\left(h_{2} \rightarrow \mathrm{DMDM}\right)+\mathrm{BR}\left(h_{2} \rightarrow h_{1} h_{1}\right)$ as a function of $m_{2}$ but only for points outside of the pattern. As expected, all those points correspond to large value of $\operatorname{BR}\left(h_{2} \rightarrow \mathrm{DMDM}\right)+\mathrm{BR}\left(h_{2} \rightarrow h_{1} h_{1}\right)$. The reason to have much fewer points outside of the pattern is that the decay $h_{2} \rightarrow$ DMDM has to be allowed while the range of

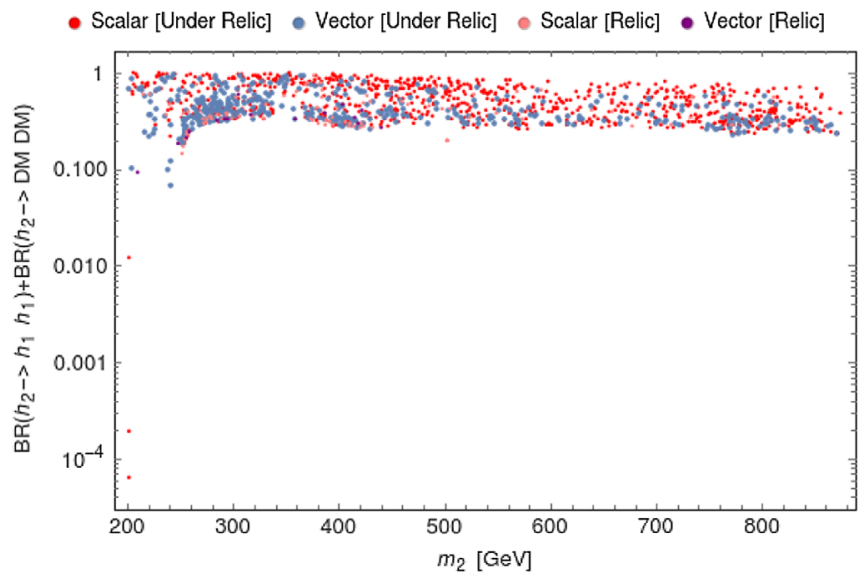

FIG. 10. $\mathrm{BR}\left(h_{2} \rightarrow h_{1} h_{1}\right)+\mathrm{BR}\left(h_{2} \rightarrow \mathrm{DMDM}\right) \quad$ vs $m_{2}$ for points that survive the bounds coming from heavy resonances and in particular $\sigma\left(p p(g g) \rightarrow h_{2} \rightarrow Z Z\right)$ with still large values $\sin \alpha$. Only points located outside of the pattern in the right panel of Fig. 9 are shown.

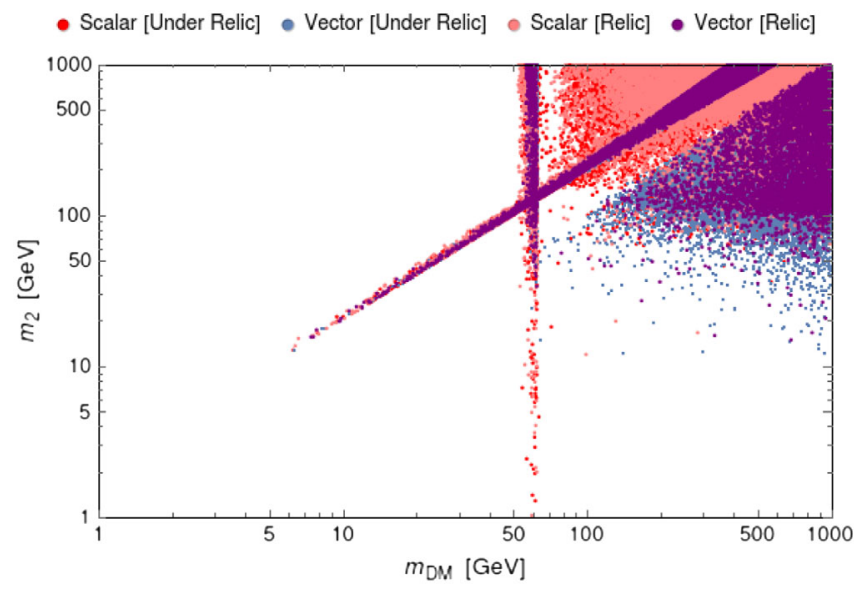

FIG. 11. Second Higgs mass $\left(m_{2}\right)$ as a function of the DM mass $\left(m_{\mathrm{DM}}\right)$.

variation of $m_{2}$ and of the $m_{\mathrm{DM}}$ is the same. That eliminates $3 / 4$ of points in the considered region.

Finally, one can clearly see the result of the searches for $h_{2} \rightarrow h_{1} h_{1}$ close to the cross section threshold and also the much harder bound for $m_{2}<m_{1} / 2$. Regarding the comparison of the two models we again see no difference and the same can be said for the projection in the $\left(\sin \alpha, m_{2}\right)$ plane.

In Fig. 11 we show $m_{2}$ as a function of the DM mass. This is a projection of the parameter space where a clear difference between the two models can be seen. There are two bands where the models coexist, close to $m_{\mathrm{DM}} \simeq m_{1} / 2$ and to $m_{2} \sim 2 \cdot m_{\mathrm{DM}}$. The explanation for the band structure could be easily guessed; these are the two resonances $h_{1}$ and $h_{2}$, respectively. In those regions, the kinematical enhancement by a resonance must be compensated by suppressed couplings that govern DM annihilation in the early Universe. This mechanism is nearly the same in both models. However, as seen from the figure there are two distinct regions above and below $m_{2}=2 \cdot m_{\mathrm{DM}}$ where only the scalar model survives. Hence, there are pairs of values $\left(m_{2}, m_{X}\right)$ that if hinted at the LHC will allow to exclude the vector model in favor of the scalar one. The reverse is not true as can be seen from the figure. The absence of VDM points in those regions is clarified in Fig. 12, where a large suppression of the cross section for scalar DM-nucleon scattering relative to the vector model one can be seen. In fact, a large portion of the parameter space of the VDM is excluded because they are above the Xenon1T bound. Therefore for a given $m_{\mathrm{DM}}$ there exist $m_{2}$ large enough to be excluded by the Xenon1T bound. On the other hand, for the SDM, even including one-loop corrections, ${ }^{3}$ all points are below the Xenon1T line. In order to have a clear picture

\footnotetext{
${ }^{3}$ Hereafter, in this context, we are referring to the estimate of the upper bound for one-loop radiative corrections as given in (2.22).
} 


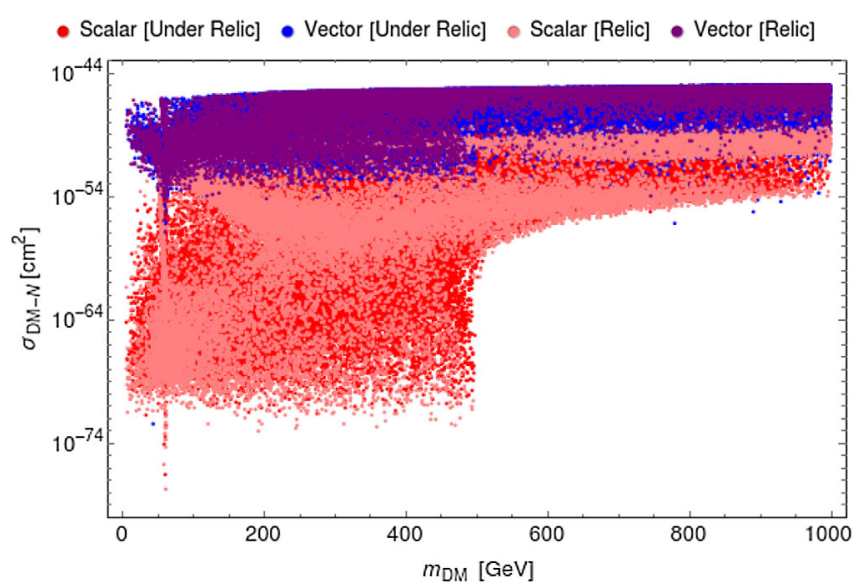

FIG. 12. DM-nucleon cross section as a function of the DM mass. Scalar DM-nucleon nucleon cross section is computed at one-loop level. The latest results from Xenon1T are shown as the solid line that makes the upper edge of the plot.

of what happens for the SDM we should compare the effect of one-loop vs tree-level result. This is shown in Fig. 13 where in the left panel we show the result for the tree-level cross section and in the right panel we show the one-loop result using Eq. (2.22). At tree-level the cross section are more than orders of magnitude below the Xenon1T line. This is due to the nature of the scalar DM coupling to the Higgs bosons for which a detailed account is given in the Appendix B. The inclusion of the one-loop contributions for the SDM increases the maximum values of the cross section by roughly ten orders of magnitude. Still only a few points are close to Xenon1T represented by the solid line (the upper edge) in the plots. Therefore, the SDM is still not affected by the direct constraints even with the one-loop corrections. Note that the points with maximally suppressed cross section correspond to $h_{2}$ resonances scattered in the range $1 \mathrm{GeV} \lesssim m_{\mathrm{DM}} \lesssim 500 \mathrm{GeV}$.

Finally, we show in Fig. 14 thermal average DM annihilation cross section into the SM times velocity (at

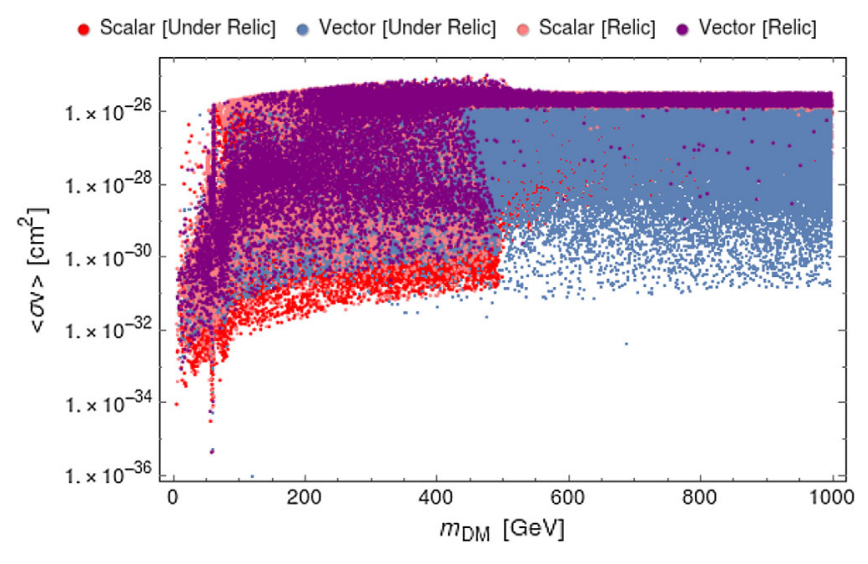

FIG. 14. Thermal average DM annihilation cross section (into the SM) times velocity (at zero temperature) vs DM mass.

zero temperature) vs DM mass. Contrary to the direct bound, the indirect bound affects both the SDM and the VDM. Although the density of points varies, the fact is that there are no major differences between the two models. Furthermore the allowed points for both models span a very large range of cross sections and therefore will most probably not be excluded in the near future.

\section{SUMMARY AND CONCLUSIONS}

In this paper we have compared scalar and vector dark matter models, both originating from the extension of the SM with an extra complex scalar $S$ and $U(1)_{X}$ symmetry. In the first model, the global $U(1)_{X}$ is softly broken by the term that generates the mass of the DM candidate which otherwise remains a massless Goldstone boson. In the second case, the $U(1)_{X}$ is local and broken spontaneously therefore this massless mode contributes, within the Higgs mechanism, to the massive vector DM particle.

We have investigated the possibility to differentiate the models by measuring the energy distribution of $Z$
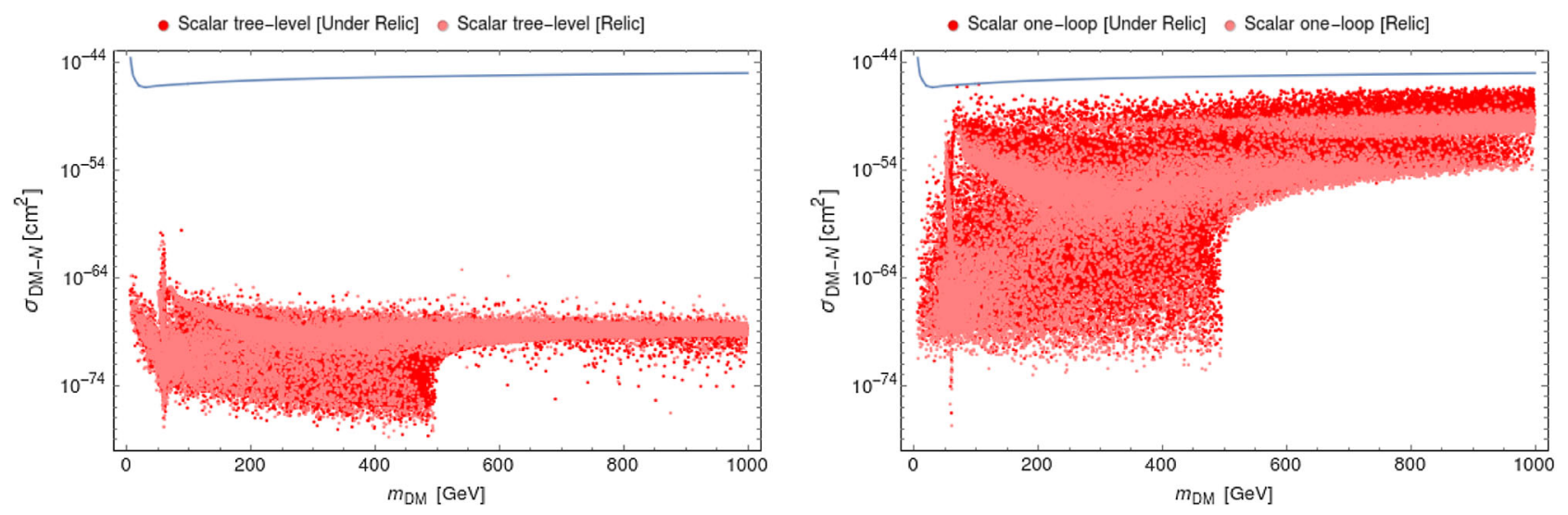

FIG. 13. Scalar DM-nucleon cross section as a function of the DM mass $\left(m_{\mathrm{DM}}=m_{A}\right)$ with the latest result from Xenon1T and relic abundance within $5 \sigma$ of experimental value. 
bosons at the ILC in the process $e^{+} e^{-} \rightarrow Z+\mathrm{DM}$. The final conclusion requires a dedicated experimental analysis which takes into account the full background and experimental details of the collider and the detector, a task which is far beyond the scope of this project. However, theoretical predictions show that there are regions in the $\left(m_{\mathrm{DM}}, m_{2}\right)$ space for which the total cross section predicted within the SDM is nearly 50\% larger than the one for the VDM, so that in those regions, future electron-positron colliders such as the ILC, CLIC or CEPC are likely to be a helpful tool in disentangling the two models. Unfortunately, the standard model $Z \nu_{l} \bar{\nu}_{l}$ final sates constitute background of considerable magnitude, making $e^{+} e^{-}$collider search for DM very challenging.

We have shown that the direct detection is efficiently suppressed in the SDM model, $\sigma_{\mathrm{DM}-N} \propto v_{A}^{4}$, as a consequence of $A$ being a pseudo-Goldstone boson. The inclusion of one-loop corrections in the direct detection cross section increases its maximum values by roughly ten orders of magnitude. Still, the bounds on direct detection do not affect the SDM.

We have determined regions in the $\left(m_{\mathrm{DM}}, m_{2}\right)$ space that are excluded for the VDM while being allowed for the SDM. If future measurements point to those regions, the VDM will not be a viable option for DM. Those regions are excluded in the VDM since the DM-nucleon scattering in this case is not particularly suppressed and therefore consistency with Xenon1T eliminates a substantial part of the VDM parameter space. In the SDM the scattering is naturally very much suppressed, and the mechanism of the suppression has been explained in a more general context.

\section{ACKNOWLEDGMENTS}

We thank Pyungwon Ko for informing us about the paper [32] by Gross, Lebedev and Toma. This work is supported in part by the National Science Centre (Poland), research Projects No. 2014/15/B/ST2/00108, 2017/25/B/ST2/00191 and 2017/25/N/ST2/01312 and a HARMONIA project under Contract No. UMO-2015/18/M/ST2/00518.

\section{APPENDIX A: GOLDSTONE-BOSON-HIGGS- BOSON COUPLING INA LINEAR FORMALISM}

In order to gain a better understanding of the cancellation observed in Sec. II A we derive the coupling between two Goldstone bosons and a Higgs boson in a slight more general context. In this Appendix we adopt the linear formalism.

Assume that the potential is composed by an invariant part, $V_{\text {inv }}$, and a softly breaking part $V_{\text {soft }}$, under certain symmetry transformation $G$

$$
\phi_{i} \rightarrow \phi_{i}+\delta \phi_{i}=\phi_{i}+i \theta^{a} T_{i j}^{a} \phi_{j},
$$

where $T^{a}$ are the generators of the Lie algebra of the group $G$ and $\theta^{a}$ are the corresponding parameters. So that

$$
\delta V=\frac{\partial V}{\partial \phi_{i}} \delta \phi_{i}=\frac{\partial V_{\text {soft }}}{\partial \phi_{i}} \delta \phi_{i}
$$

with $V=V_{\text {inv }}+V_{\text {soft }}$. We assume $\phi_{i}$ are real fields. Explicitly one can write

$$
\frac{\partial V}{\partial \phi_{i}} \theta^{a} T_{i j}^{a} \phi_{j}=\frac{\partial V_{\text {soft }}}{\partial \phi_{i}} \theta^{a} T_{i j}^{a} \phi_{j}
$$

Differentiating twice with respect to $\phi_{k}$ and $\phi_{l}$ and evaluating the final expression at a minimum $\phi_{n}=\left\langle\phi_{n}\right\rangle=$ $v_{n}$ of the full theory, i.e., for $V=V_{\text {inv }}+V_{\text {soft }}$, one obtains

$$
\begin{aligned}
V_{l k i} \theta^{a} T_{i j}^{a} v_{j}+\left\{M_{k i}^{2} \theta^{a} T_{i l}^{a}+(k \leftrightarrow l)\right\} \\
=\left.\frac{\partial^{3} V_{\text {soft }}}{\partial \phi_{l} \partial \phi_{k} \partial \phi_{i}}\right|_{\phi_{n}=v_{n}} \theta^{a} T_{i j}^{a} v_{j} \\
\quad+\left\{\left.\frac{\partial^{2} V_{\text {soft }}}{\partial \phi_{k} \partial \phi_{i}}\right|_{\phi_{n}=v_{n}} \theta^{a} T_{i l}^{a}+(k \leftrightarrow l)\right\},
\end{aligned}
$$

where

$$
\begin{aligned}
& \left.V_{l k i} \equiv \frac{\partial^{3} V}{\partial \phi_{l} \partial \phi_{k} \partial \phi_{i}}\right|_{\phi_{n}=v_{n}} \text { and } \\
& \left.M_{k i}^{2} \equiv \frac{\partial^{2} V}{\partial \phi_{k} \partial \phi_{i}}\right|_{\phi_{n}=v_{n}}
\end{aligned}
$$

We shall specialize to the case of a complex singlet $S$ charged under a $U(1)$ symmetry

$$
\begin{aligned}
\phi & =\left(\begin{array}{c}
\phi_{1} \\
\vdots \\
\phi_{N-2} \\
s \equiv \frac{\mathrm{Re} S}{\sqrt{2}} \\
a \equiv \frac{\mathrm{Im} S}{\sqrt{2}}
\end{array}\right) \quad v=\langle\phi\rangle=\left(\begin{array}{c}
v_{1} \\
\vdots \\
v_{N-2} \\
v_{S} \\
0
\end{array}\right) \\
M^{2} & =\left(\begin{array}{cccc}
M_{1,1}^{2} & \cdots & M_{1, N-1}^{2} & 0 \\
\vdots & \ddots & \vdots & \vdots \\
M_{N-1,1}^{2} & \cdots & M_{N-1, N-1}^{2} & 0 \\
0 & \cdots & 0 & m_{a}^{2}
\end{array}\right)
\end{aligned}
$$

Note that the mass matrix $M^{2}$ is, in general, nondiagonal. Since we assume invariance under $S \rightarrow S^{*}$, there is no mixing between $\operatorname{Im} S$ and other states in the mass matrix if $\langle a\rangle=0$. Since the $U(1)$ is softly broken the $a$ mass could be nonzero, i.e., a pseudo-Goldstone boson. 
The $U(1)$ generator in this basis reads

$$
T=\left(\begin{array}{cccc}
0 & \cdots & 0 & 0 \\
\vdots & \ddots & \vdots & \vdots \\
0 & \cdots & 0 & i \\
0 & \cdots & -i & 0
\end{array}\right) .
$$

In other words

$$
T_{i l}=i\left(\delta_{i, N-1} \delta_{l, N}-\delta_{i, N} \delta_{l, N-1}\right),
$$

so that

$$
\begin{aligned}
M_{k i}^{2} T_{i l} & =i\left(M_{k, N-1}^{2} \delta_{l, N}-M_{k, N}^{2} \delta_{l, N-1}\right), \quad \text { and } \\
T_{i j} v_{j} & =-i \delta_{i, N} v_{S} .
\end{aligned}
$$

Replacing the above in (A4) and choosing the $V_{l N N}$ component one finds

$$
\begin{aligned}
V_{l N N} v_{S}= & M_{l, N-1}^{2}-\delta M_{l, N-1}^{2} \\
& -\left(m_{a}^{2}-\delta M_{N, N}^{2}\right) \delta_{l, N-1}+\left.\frac{\partial^{3} V_{\text {soft }}}{\partial \phi_{l} \partial \phi_{N} \partial \phi_{N}}\right|_{\phi_{n}=v_{n}} v_{S},
\end{aligned}
$$

where

$$
\left.\delta M_{k, i}^{2} \equiv \frac{\partial^{2} V_{\text {soft }}}{\partial \phi_{k} \partial \phi_{i}}\right|_{\phi_{n}=v_{n}} .
$$

Note that if $V_{\text {soft }} \neq 0$ then $m_{a}^{2}$ receives contributions from the symmetric part of the potential as well ${ }^{4}$ and therefore

$\left.m_{a}^{2} \equiv \frac{\partial^{2} V}{\partial \phi_{N} \partial \phi_{N}}\right|_{\phi_{n}=v_{n}} \neq\left.\frac{\partial^{2} V_{\text {soft }}}{\partial \phi_{N} \partial \phi_{N}}\right|_{\phi_{n}=v_{n}} \equiv \delta M_{N, N}^{2}$

In the symmetric limit of $V_{\text {soft }} \rightarrow 0$ one obtains $V_{l N N} v_{S}=$ $M_{l, N-1}^{2}$. Note that the contribution $m_{a}^{2}-\delta M_{N, N}^{2}$ might be written also in the following way

$$
m_{a}^{2}-\delta M_{N, N}^{2}=\left.\frac{\partial^{2} V_{\mathrm{inv}}}{\partial \phi_{N} \partial \phi_{N}}\right|_{\phi_{n}=v_{n}},
$$

where $v_{i}$ in the vacuum of the full theory, i.e., for $V=V_{\text {inv }}+V_{\text {soft }}$.

The mass matrix $M^{2}$ could be diagonalized by an orthogonal rotation $R$ as follows

$$
M^{2}=R \mathcal{M}^{2} R^{T}
$$

\footnotetext{
${ }^{4}$ Of course, those contributions vanish in the limit $V_{\text {soft }} \rightarrow 0$.
}

where $\mathcal{M}^{2}$ is the diagonal matrix. The mass eigenstates are $\varphi=R^{T} \phi$. The rotation matrix is of the form

$$
R=\left(\begin{array}{cccc}
R_{1,1} & \cdots & R_{1, N-1} & 0 \\
\vdots & \ddots & \vdots & \vdots \\
R_{N-1,1} & \cdots & R_{N-1, N-1} & 0 \\
0 & \cdots & 0 & 1
\end{array}\right)
$$

The cubic coupling that is relevant for us could be written in terms of the mass eigenstates as follows

$$
V=\cdots+V_{l k i} R_{l l^{\prime}} \varphi_{l^{\prime}} R_{k k^{\prime}} \varphi_{k^{\prime}} R_{i i^{\prime}} \varphi_{i^{\prime}}+\cdots
$$

We are interested in the $V_{l N N}$ vertex and therefore we choose $k^{\prime}=i^{\prime}=N$. We also limit ourself to $l^{\prime} \neq N$. Since $R_{k, N}=\delta_{k, N}$ and $R_{i, N}=\delta_{i, N}$,

$$
V=\cdots+V_{l N N} R_{l l^{\prime}} \varphi_{l^{\prime}} \varphi_{N} \varphi_{N}+\cdots
$$

The term $M_{l, N-1}^{2} R_{l l^{\prime}}$ from (A10) together with (A17) can be expressed by mass eigenvalues and mixing angles as $M_{l, N-1}^{2} R_{l l^{\prime}}=m_{l^{\prime}}^{2} R_{N-1, l^{\prime}}$. Then the coefficient of $\varphi_{l^{\prime}} \varphi_{N} \varphi_{N}$ (with $l^{\prime} \neq N$ ) reads

$$
\begin{aligned}
& \frac{1}{v_{S}}\left\{m_{l^{\prime}}^{2} R_{N-1, l^{\prime}}+\left[\frac{\partial^{3} V_{\text {soft }}}{\partial \phi_{l} \partial \phi_{N} \partial \phi_{N}} v_{S} R_{l, l^{\prime}}-\frac{\partial^{2} V_{\text {inv }}}{\partial \phi_{N} \partial \phi_{N}} R_{N-1, l^{\prime}}\right.\right. \\
& \left.\left.-\frac{\partial^{2} V_{\text {soft }}}{\partial \phi_{l} \partial \phi_{N-1}} R_{l, l^{\prime}}\right]\left.\right|_{\phi_{n}=v_{n}}\right\} .
\end{aligned}
$$

The above equation allows to calculate corrections to the $U(1)$-symmetric relation $V_{l^{\prime} N N}=M_{l^{\prime}, N-1}^{2} / v_{S}$ for a given symmetry-breaking potential $V_{\text {soft }}$. For instance for $V_{\text {soft }}=$ $\mu^{2}\left(S^{2}+S^{* 2}\right)$ the first term in the bracket is trivially zero while the remaining ones sum to zero

$$
\begin{aligned}
& {\left.\left[-\frac{\partial^{2} V_{\text {inv }}}{\partial \phi_{N} \partial \phi_{N}} R_{N-1, l^{\prime}}-\frac{\partial^{2} V_{\text {soft }}}{\partial \phi_{l} \partial \phi_{N-1}} R_{l, l^{\prime}}\right]\right|_{\phi_{n}=v_{n}}} \\
& \quad=\left(4 \mu^{2}-2 \mu^{2}-2 \mu^{2}\right) R_{N-1, l^{\prime}}=0
\end{aligned}
$$

That way we have reproduced the result of (2.20). It is also worthwhile to consider a linear $U(1)$ breaking, by $M^{3}\left(S+S^{*}\right) / \sqrt{2}$. In this case, even though derivatives of $V_{\text {soft }}$ do not contribute to corrections to $V_{l^{\prime} N N}=M_{l^{\prime}, N-1}^{2} / v_{S}$, the derivative of $V_{\text {inv }}$, as it is evaluated at the minimum of the full theory, does contribute:

$$
\left.\frac{\partial^{2} V_{\text {inv }}}{\partial \phi_{N} \partial \phi_{N}}\right|_{\phi_{n}=v_{n}}=-\frac{M^{3}}{v_{S}}
$$


Therefore we conclude that soft $U(1)$ breaking terms other than the quadratic ones may spoil the proportionality of the coupling to the Higgs mass squared observed in (2.20).

\section{APPENDIX B: PSEUDO-GOLDSTONE-BOSON- HIGGS-BOSON COUPLINGS IN THE NONLINEAR FORMALISM}

In this Appendix we rederive the above effective pseudoGoldstone-Higgs couplings within the nonlinear realization of the same Lagrangian. Here we write down the complex field $S$ in the following form:

$$
S=\frac{1}{\sqrt{2}}\left(v_{s}+s\right) e^{i a / v_{s}}
$$

so that the $U(1)$ symmetric part of the potential does not contain couplings involving the Goldstone boson $a$ any more. Since $a$ is odd under the $Z_{2}$ symmetry transformation $S \leftrightarrow S^{*}$, it can be an appropriate DM candidate. The only terms that $a$ appears in are the kinetic and the $U(1)$ softlybreaking terms. We will consider linear and quadratic breaking as follows:

$$
\begin{aligned}
\mathcal{L}_{a}= & \partial^{\mu} S^{*} \partial_{\mu} S-\frac{M^{3}}{\sqrt{2}}\left(S+S^{*}\right)-\mu^{2}\left(S^{2}+S^{* 2}\right) \\
= & \frac{\left(v_{s}+s\right)^{2}}{2 v_{s}^{2}} \partial^{\mu} a \partial_{\mu} a-M^{3}\left(v_{s}+s\right) \cos \left(\frac{a}{v_{s}}\right) \\
& -\mu^{2}\left(v_{s}+s\right)^{2} \cos \left(\frac{2 a}{v_{s}}\right) \\
\supset & \frac{1}{2} \partial^{\mu} a \partial_{\mu} a+\frac{1}{2}\left(4 \mu^{2}+\frac{M^{3}}{v_{s}}\right) a^{2}+\frac{s}{v_{s}} \partial^{\mu} a \partial_{\mu} a \\
& +\left(\frac{4 \mu^{2}}{v_{s}}+\frac{M^{3}}{2 v_{s}^{2}}\right) s a^{2},
\end{aligned}
$$

from which we can easily read off the pseudoscalar DM mass squared as $m_{a}^{2}=-4 \mu^{2}-M^{3} / v_{s}$, which is the same as that obtained within the linear realization of the $U(1)$ symmetry.

Now we are going to show that the pseudo-GoldstoneHiggs vertex agrees with the result obtained in the
Appendix A. We focus on the following vertex involving partial derivatives of $a$

$$
\begin{aligned}
\frac{1}{v_{s}} s \partial^{\mu} a \partial_{\mu} a & =-\frac{1}{v_{s}}\left(\partial^{\mu} s \partial_{\mu} a\right) a-\frac{1}{v_{s}} s a \square a \\
& =-\frac{1}{2 v_{s}} \partial^{\mu} s \partial_{\mu}\left(a^{2}\right)+\frac{m_{a}^{2}}{v_{s}} s a^{2} \\
& =\frac{1}{2 v_{s}}(\square s) a^{2}+\frac{m_{a}^{2}}{v_{s}} s a^{2} \\
& =\frac{1}{2 v_{s}}\left(\sin \alpha \square h_{1}+\cos \alpha \square h_{2}\right) a^{2}+\frac{m_{a}^{2}}{v_{s}} s a^{2} \\
& =-\frac{1}{2 v_{s}}\left(\sin \alpha m_{1}^{2} h_{1}+\cos \alpha m_{2}^{2} h_{2}\right) a^{2}+\frac{m_{a}^{2}}{v_{s}} s a^{2},
\end{aligned}
$$

where we have repeatedly used the integration by parts and exploited free equations of motion for $a$ and $h_{1,2}$, i.e., $\square a=-m_{a}^{2} a$ and $\square h_{i}=-m_{i}^{2} h_{i}$. By putting the final expression of Eq. (B3) into Eq. (B2), we obtain

$$
\begin{aligned}
\mathcal{L}_{a} \supset & \frac{1}{2}\left(\partial^{\mu} a \partial_{\mu} a-m_{a}^{2} a^{2}\right)-\frac{1}{2 v_{s}}\left(\sin \alpha m_{1}^{2} h_{1}+\cos \alpha m_{2}^{2} h_{2}\right) a^{2} \\
& +\frac{1}{v_{s}}\left(4 \mu^{2}+\frac{M^{3}}{2 v_{s}}+m_{a}^{2}\right) s a^{2} \\
= & \frac{1}{2}\left(\partial^{\mu} a \partial_{\mu} a-m_{a}^{2} a^{2}\right)-\frac{1}{2 v_{s}}\left(\sin \alpha m_{1}^{2} h_{1}+\cos \alpha m_{2}^{2} h_{2}\right) a^{2} \\
& -\frac{M^{3}}{2 v_{s}^{2}} s a^{2} \\
= & \frac{1}{2}\left(\partial^{\mu} a \partial_{\mu} a-m_{a}^{2} a^{2}\right)-\frac{1}{2 v_{s}}\left(\sin \alpha m_{1}^{2} h_{1}+\cos \alpha m_{2}^{2} h_{2}\right) a^{2} \\
& -\frac{M^{3}}{2 v_{s}^{2}}\left(\sin \alpha h_{1}+\cos \alpha h_{2}\right) a^{2} .
\end{aligned}
$$

So indeed, the coupling is the same as obtained in the Appendix A and in Sec. II A.
[1] G. Aad et al. (ATLAS Collaboration), Combined search for the standard model Higgs boson using up to $4.9 \mathrm{fb}^{-1}$ of $p p$ collision data at $\sqrt{s}=7 \mathrm{TeV}$ with the ATLAS detector at the LHC, Phys. Lett. B 710, 49 (2012).

[2] S. Chatrchyan et al. (CMS Collaboration), Combined results of searches for the standard model Higgs boson in $p p$ collisions at $\sqrt{s}=7 \mathrm{TeV}$, Phys. Lett. B 710, 26 (2012).
[3] V. Silveira and A. Zee, Scalar phantoms, Phys. Lett. 161B, 136 (1985).

[4] J. McDonald, Gauge singlet scalars as cold dark matter, Phys. Rev. D 50, 3637 (1994).

[5] C. P. Burgess, M. Pospelov, and T. ter Veldhuis, The minimal model of nonbaryonic dark matter: A singlet scalar, Nucl. Phys. B619, 709 (2001). 
[6] M. C. Bento, O. Bertolami, R. Rosenfeld, and L. Teodoro, Selfinteracting dark matter and invisibly decaying Higgs, Phys. Rev. D 62, 041302 (2000).

[7] M. Gonderinger, H. Lim, and M. J. Ramsey-Musolf, Complex scalar singlet dark matter: Vacuum stability and phenomenology, Phys. Rev. D 86, 043511 (2012).

[8] E. Gabrielli, M. Heikinheimo, K. Kannike, A. Racioppi, M. Raidal, and C. Spethmann, Towards completing the standard model: Vacuum stability, EWSB and dark matter, Phys. Rev. D 89, 015017 (2014).

[9] T. Hambye, Hidden vector dark matter, J. High Energy Phys. 01 (2009) 028.

[10] O. Lebedev, H. M. Lee, and Y. Mambrini, Vector Higgsportal dark matter and the invisible Higgs, Phys. Lett. B 707, 570 (2012).

[11] Y. Farzan and A. R. Akbarieh, VDM: A model for vector dark matter, J. Cosmol. Astropart. Phys. 10 (2012) 026.

[12] S. Baek, P. Ko, W.-I. Park, and E. Senaha, Higgs portal vector dark matter: Revisited, J. High Energy Phys. 05 (2013) 036.

[13] S. Baek, P. Ko, and W.-I. Park, Invisible Higgs decay width vs dark matter direct detection cross section in Higgs portal dark matter models, Phys. Rev. D 90, 055014 (2014).

[14] M. Duch, B. Grzadkowski, and M. McGarrie, A stable Higgs portal with vector dark matter, J. High Energy Phys. 09 (2015) 162.

[15] S. Profumo, M. J. Ramsey-Musolf, C. L. Wainwright, and P. Winslow, Singlet-catalyzed electroweak phase transitions and precision Higgs boson studies, Phys. Rev. D 91, 035018 (2015).

[16] V. Barger, D. J. H. Chung, A. J. Long, and L.-T. Wang, Strongly first order phase transitions near an enhanced discrete symmetry point, Phys. Lett. B 710, 1 (2012).

[17] J. R. Espinosa, T. Konstandin, and F. Riva, Strong electroweak phase transitions in the standard model with a singlet, Nucl. Phys. B854, 592 (2012).

[18] J. McDonald, Electroweak baryogenesis and dark matter via a gauge singlet scalar, Phys. Lett. B 323, 339 (1994).

[19] G. C. Branco, D. Delepine, D. Emmanuel-Costa, and F. R. Gonzalez, Electroweak baryogenesis in the presence of an isosinglet quark, Phys. Lett. B 442, 229 (1998).

[20] V. Barger, P. Langacker, M. McCaskey, M. Ramsey-Musolf, and G. Shaughnessy, Complex singlet extension of the standard model, Phys. Rev. D 79, 015018 (2009).

[21] M. Jiang, L. Bian, W. Huang, and J. Shu, Impact of a compl ex singlet: Electroweak baryogenesis and dark matter, Phys. Rev. D 93, 065032 (2016).

[22] W. Chao, $C P$ violation at the finite temperature, arXiv:1706 .01041 .

[23] W. Chao, First order electroweak phase transition triggered by the Higgs portal vector dark matter, Phys. Rev. D 92, 015025 (2015).

[24] B. Patt and F. Wilczek, Higgs-field portal into hidden sectors, arXiv:hep-ph/0605188.

[25] G. Degrassi, S. Di Vita, J. Elias-Miro, J. R. Espinosa, G. F. Giudice, G. Isidori, and A. Strumia, Higgs mass and vacuum stability in the standard model at NNLO, J. High Energy Phys. 08 (2012) 098.
[26] S. Alekhin, A. Djouadi, and S. Moch, The top quark and Higgs boson masses and the stability of the electroweak vacuum, Phys. Lett. B 716, 214 (2012).

[27] R. Costa, A. P. Morais, M. O. P. Sampaio, and R. Santos, Two-loop stability of a complex singlet extended standard model, Phys. Rev. D 92, 025024 (2015).

[28] R. Coimbra, M. O. P. Sampaio, and R. Santos, ScannerS: Constraining the phase diagram of a complex scalar singlet at the LHC, Eur. Phys. J. C 73, 2428 (2013).

[29] S. Pokorski, Gauge Field Theories (Cambridge University Press, Cambridge, England, 2005).

[30] V. Barger, M. McCaskey, and G. Shaughnessy, Complex scalar dark matter vis-à-vis CoGeNT, DAMA/LIBRA and XENON100, Phys. Rev. D 82, 035019 (2010).

[31] J. Liu, X.-P. Wang, and F. Yu, A tale of two portals: Testing light, hidden new physics at future $e^{+} e^{-}$colliders, J. High Energy Phys. 06 (2017) 077.

[32] C. Gross, O. Lebedev, and T. Toma, Cancellation Mechanism for Dark-Matter Nucleon Interaction, Phys. Rev. Lett. 119, 191801 (2017).

[33] C. Patrignani et al. (Particle Data Group), Review of particle physics, Chin. Phys. C 40, 100001 (2016).

[34] M. Duch, B. Grzadkowski, and D. Huang, Strongly selfinteracting vector dark matter via freeze-in, J. High Energy Phys. 01 (2018) 020.

[35] A. Birkedal, K. Matchev, and M. Perelstein, Dark matter at colliders: A model independent approach, Phys. Rev. D 70, 077701 (2004).

[36] J. L. Feng, S. Su, and F. Takayama, Lower Limit on Dark Matter Production at the Large Hadron Collider, Phys. Rev. Lett. 96, 151802 (2006).

[37] P. Konar, K. Kong, K. T. Matchev, and M. Perelstein, Shedding light on the dark sector with direct WIMP production, New J. Phys. 11, 105004 (2009).

[38] J. Goodman, M. Ibe, A. Rajaraman, W. Shepherd, T. M. P. Tait, and H.-B. Yu, Constraints on dark matter from colliders, Phys. Rev. D 82, 116010 (2010).

[39] P. J. Fox, R. Harnik, J. Kopp, and Y. Tsai, Missing energy signatures of dark matter at the LHC, Phys. Rev. D 85, 056011 (2012).

[40] J. Kumar, D. Marfatia, and D. Yaylali, Vector dark matter at the LHC, Phys. Rev. D 92, 095027 (2015).

[41] D. Alves (LHC New Physics Working Group), Simplified models for LHC new physics searches, J. Phys. G 39, 105005 (2012).

[42] D. Abercrombie et al., Dark matter benchmark models for early LHC run-2 searches: Report of the ATLAS/CMS dark matter forum, arXiv:1507.00966.

[43] M. R. Buckley, D. Feld, and D. Goncalves, Scalar simplified models for dark matter, Phys. Rev. D 91, 015017 (2015).

[44] J. Abdallah et al., Simplified models for dark matter searches at the LHC, Phys. Dark Universe 9-10, 8 (2015).

[45] T. Abe et al. (LHC Dark Matter Working Group), LHC Dark matter working group: Next-generation spin-0 dark matter models, arXiv:1810.09420.

[46] C.-H. Chen and T. Nomura, Searching for vector dark matter via Higgs portal at the LHC, Phys. Rev. D 93, 074019 (2016). 
[47] S. Baek, P. Ko, M. Park, W.-I. Park, and C. Yu, Beyond the dark matter effective field theory and a simplified model approach at colliders, Phys. Lett. B 756, 289 (2016).

[48] P. Ko and J. Li, Interference effects of two scalar boson propagators on the LHC search for the singlet fermion DM, Phys. Lett. B 765, 53 (2017).

[49] E. A. Baltz, M. Battaglia, M. E. Peskin, and T. Wizansky, Determination of dark matter properties at high-energy colliders, Phys. Rev. D 74, 103521 (2006).

[50] H. Dreiner, M. Huck, M. Kramer, D. Schmeier, and J. Tattersall, Illuminating dark matter at the ILC, Phys. Rev. D 87, 075015 (2013).

[51] Z.-H. Yu, X.-J. Bi, Q.-S. Yan, and P.-F. Yin, Dark matter searches in the mono- $Z$ channel at high energy $e^{+} e^{-}$ colliders, Phys. Rev. D 90, 055010 (2014).

[52] N. Wan, M. Song, G. Li, W.-G. Ma, R.-Y. Zhang, and J.-Y. Guo, Searching for dark matter via mono- $Z$ boson production at the ILC, Eur. Phys. J. C 74, 3219 (2014).

[53] P. Ko and H. Yokoya, Search for Higgs portal DM at the ILC, J. High Energy Phys. 08 (2016) 109.

[54] S. Dutta, D. Sachdeva, and B. Rawat, Signals of leptophilic dark matter at the ILC, Eur. Phys. J. C 77, 639 (2017).

[55] T. Kamon, P. Ko, and J. Li, Characterizing Higgs portal dark matter models at the ILC, Eur. Phys. J. C 77, 652 (2017).

[56] K. Fujii et al., The role of positron polarization for the inital $250 \mathrm{GeV}$ stage of the international linear collider, arXiv: 1801.02840.

[57] D. Dannheim, P. Lebrun, L. Linssen, D. Schulte, F. Simon, S. Stapnes, N. Toge, H. Weerts, and J. Wells, CLIC $e^{+} e^{-}$ linear collider studies, arXiv:1208.1402.

[58] CEPC Study Group, CEPC conceptual design report: Volume 2-Physics \& detector, arXiv:1811.10545.

[59] C. Adolphsen, M. Barone, B. Barish, K. Buesser, P. Burrows, J. Carwardine, J. Clark, H. Mainaud Durand, G. Dugan, E. Elsen et al., The international linear collider technical design report - volume 3.II: Accelerator baseline design, arXiv:1306.6328.

[60] A. Belyaev, N. D. Christensen, and A. Pukhov, CalcHEP 3.4 for collider physics within and beyond the standard model, Comput. Phys. Commun. 184, 1729 (2013).

[61] M. Tanabashi et al. (Particle Data Group), Review of particle physics, Phys. Rev. D 98, 030001 (2018).

[62] L. Linssen, A. Miyamoto, M. Stanitzki, and H. Weerts, Physics and detectors at CLIC: CLIC conceptual design report, arXiv:1202.5940.

[63] R. Costa, M. Mhlleitner, M. O. P. Sampaio, and R. Santos, Singlet extensions of the standard model at LHC run 2: Benchmarks and comparison with the NMSSM, J. High Energy Phys. 06 (2016) 034.

[64] G. Aad et al. (ATLAS and CMS Collaboration), Combined Measurement of the Higgs Boson Mass in $p p$ Collisions at $\sqrt{s}=7$ and $8 \mathrm{TeV}$ with the ATLAS and CMS Experiments, Phys. Rev. Lett. 114, 191803 (2015).

[65] M.E. Peskin and T. Takeuchi, Estimation of oblique electroweak corrections, Phys. Rev. D 46, 381 (1992).

[66] W. Grimus, L. Lavoura, O. M. Ogreid, and P. Osland, The oblique parameters in multi-Higgs-doublet models, Nucl. Phys. B801, 81 (2008).
[67] P. Bechtle, O. Brein, S. Heinemeyer, G. Weiglein, and K. E. Williams, HiggsBounds: confronting arbitrary Higgs sectors with exclusion bounds from LEP and the Tevatron, Comput. Phys. Commun. 181, 138 (2010).

[68] A. Djouadi, J. Kalinowski, and M. Spira, HDECAY: A program for Higgs boson decays in the standard model and its supersymmetric extension, Comput. Phys. Commun. 108, 56 (1998).

[69] J. M. Butterworth et al., The Tools And Monte Carlo Working Group Summary Report From The Les Houches 2009 Workshop On Tev Colliders, arXiv:1003.1643.

[70] M. Aaboud et al. (ATLAS Collaboration), Search for heavy $Z Z$ resonances in the $\ell^{+} \ell^{-} \ell^{+} \ell^{-}$and $\ell^{+} \ell^{-} \nu \bar{\nu}$ final states using proton proton collisions at $\sqrt{s}=13 \mathrm{TeV}$ with the ATLAS detector, Eur. Phys. J. C 78, 293 (2018).

[71] M. Aaboud et al. (ATLAS Collaboration), Search for heavy resonances decaying into $W W$ in the $e \nu \mu \nu$ final state in $p p$ collisions at $\sqrt{s}=13 \mathrm{TeV}$ with the ATLAS detector, Eur. Phys. J. C 78, 24 (2018).

[72] M. Aaboud et al. (ATLAS Collaboration), Combination of searches for heavy resonances decaying into bosonic and leptonic final states using $36 \mathrm{fb}^{-1}$ of proton-proton collision data at $\sqrt{s}=13 \mathrm{TeV}$ with the ATLAS detector, Phys. Rev. D 98, 052008 (2018).

[73] M. Aaboud et al. (ATLAS Collaboration), Search for pair production of Higgs bosons in the $b \bar{b} b \bar{b}$ final state using proton-proton collisions at $\sqrt{s}=13 \mathrm{TeV}$ with the ATLAS detector, arXiv:1804.06174.

[74] M. Aaboud et al. (ATLAS Collaboration), Search for Resonant and Nonresonant Higgs Boson Pair Production in the $b \bar{b} \tau^{+} \tau^{-}$Decay Channel in $p p$ Collisions at $\sqrt{s}=$ $13 \mathrm{TeV}$ with the ATLAS Detector, Phys. Rev. Lett. 121, 191801 (2018).

[75] G. Belanger, F. Boudjema, A. Pukhov, and A. Semenov, micrOMEGAs_3: A program for calculating dark matter observables, Comput. Phys. Commun. 185, 960 (2014).

[76] P. A. R. Ade et al. (Planck Collaboration), Planck 2015 results. XIII. Cosmological parameters, Astron. Astrophys. 594, A13 (2016).

[77] D. S. Akerib et al. (LUX Collaboration), Results from a Search for Dark Matter in the Complete LUX Exposure, Phys. Rev. Lett. 118, 021303 (2017).

[78] X. Cui et al. (PandaX-II Collaboration), Dark Matter Results From 54-Ton-Day Exposure of PandaX-II Experiment, Phys. Rev. Lett. 119, 181302 (2017).

[79] E. Aprile et al. (XENON Collaboration), First Dark Matter Search Results from the XENON1T Experiment, Phys. Rev. Lett. 119, 181301 (2017).

[80] E. Aprile et al. (XENON Collaboration), Dark Matter Search Results from a One Tonne $\times$ Year Exposure of XENON1T, Phys. Rev. Lett. 121, 111302 (2018).

[81] G. Angloher et al. (CRESST Collaboration), Results on light dark matter particles with a low-threshold CRESST-II detector, Eur. Phys. J. C 76, 25 (2016).

[82] R. Agnese et al. (SuperCDMS Collaboration), New Results from the Search for Low-Mass Weakly Interacting Massive Particles with the CDMS Low Ionization Threshold Experiment, Phys. Rev. Lett. 116, 071301 (2016). 
[83] M. Ackermann et al. (Fermi-LAT Collaboration), Searching for Dark Matter Annihilation from Milky Way Dwarf Spheroidal Galaxies with Six Years of Fermi Large Area Telescope Data, Phys. Rev. Lett. 115, 231301 (2015).

[84] M. Aguilar et al. (AMS Collaboration), Precision Measurement of the $\left(e^{+}+e^{-}\right)$Flux in Primary Cosmic Rays from $0.5 \mathrm{GeV}$ to $1 \mathrm{TeV}$ with the Alpha Magnetic Spectrometer on the International Space Station, Phys. Rev. Lett. 113, 221102 (2014).
[85] L. Accardo et al. (AMS Collaboration), High Statistics Measurement of the Positron Fraction in Primary Cosmic Rays of $0.5500 \mathrm{GeV}$ with the Alpha Magnetic Spectrometer on the International Space Station, Phys. Rev. Lett. 113, 121101 (2014).

[86] G. Elor, N. L. Rodd, T. R. Slatyer, and W. Xue, Modelindependent indirect detection constraints on hidden sector dark matter, J. Cosmol. Astropart. Phys. 06 (2016) 024. 